\title{
Novel Radio Link Buffer Management Schemes for End-user Multi-class Traffic in High Speed Downlink Packet Access Networks.
}

SULEIMAN YERIMA and KHALID AL-BEGAIN

\section{Postprint}

Full article information:

Yerima, S. Y., \& Al-Begain, K. (2011). Novel Radio Link Buffer Management Schemes for End-User Multi-class Traffic in High Speed Packet Access Networks. Wireless Personal Communications, 61(2), 349-382. 


\title{
Novel Radio Link Buffer Management Schemes for End-user Multi-class Traffic in High Speed Downlink Packet Access Networks.
}

\author{
SULEIMAN YERIMA and KHALID AL-BEGAIN \\ Integrated Communications Research Centre, Faculty of Advanced Technology, University of Glamorgan, \\ CF37 1SQ, Pontypridd, UK \\ E-mail: syerima@glam.ac.uk; kbegain@glam.ac.uk
}

Tel: 01443483612

Fax: 01443654087

\begin{abstract}
High Speed Downlink Packet Access (HSDPA) is a $3.5 \mathrm{G}$ wireless system introduced to improve downlink packet data and multimedia services support in 3G Universal Mobile Telecommunication Systems (UMTS). HSDPA standards support end-user multi-class sessions comprising parallel flows with diverse Quality of Service (QoS) requirements, such as real-time (RT) voice or video streaming concurrent with non real-time (NRT) data service being transmitted to the same user, with differentiated queuing at the radio link interface i.e. Node B. Hence, in this paper we present and evaluate novel HSDPA radio link buffer management schemes for QoS control of multimedia traffic comprising concurrent RT and NRT flows in the same HSDPA end-user session. The new buffer management schemes - Enhanced Time Space Priority (E-TSP) and Dynamic Time Space Priority (D-TSP) - are designed to improve radio link and network resource utilization as well as optimize end-to-end QoS performance of both RT and NRT flows in the end-user session. Both schemes are based on a Time-Space Priority (TSP) queuing system, which provides joint delay and loss differentiation between the flows by queuing (partially) loss tolerant RT flow packets for higher transmission priority but with restricted access to the buffer space, whilst allowing unlimited access to the buffer space for delay-tolerant NRT flow but with queuing for lower transmission priority. Experiments by means of extensive system-level HSDPA simulations demonstrates that with the proposed TSP-based radio link buffer management schemes, significant end-to-end QoS performance gains accrue to end-user traffic with simultaneous RT and NRT flows, in addition to improved resource utilization in the radio access network.
\end{abstract}

Keywords: HSDPA, buffer management, QoS, multimedia, queuing theory, performance evaluation 


\section{INTRODUCTION}

Universal Mobile Telecommunications Systems (UMTS) is a Third Generation mobile system designed for enhanced person-toperson communications, access to information and services on public and private networks, and new flexible communication capabilities [1]. The third generation partnership project (3GPP), standardized High Speed Downlink Packet Access (HSDPA) [2] as a set of technological enhancements to UMTS, whose combination improves network capacity, spectrum efficiency, and peak data rates up to $14.4 \mathrm{Mbps}$ for downlink packet data traffic. The availability of higher data rates will enable developers to create content rich multimedia applications, typically consisting of a number of classes of media or data, with different Quality of Service (QoS) requirements being concurrently downloaded to a single user [3]. Furthermore, higher data rate packet data support as well as proliferation of advanced mobile terminals will enable mobile broadband multimedia sessions characterised by multiple flows being transmitted in parallel to the same end-user; for instance, VoIP communication during an FTP download or a streaming video session concurrent with data download to the same terminal. Hence, in order to provide optimum end-to-end QoS guarantees for the multiple flows present in such mobile multimedia sessions, the system must support mechanisms to enable session/connection level (i.e. intra-user) traffic differentiation, priority handling, and QoS management especially at the radio link which poses a severe bottleneck to end-to-end communication.

In HSDPA, the base station (i.e. Node B) is responsible for scheduling data transmission to multiple end-user terminals in a cell on a shared downlink channel. Similarly, physical layer data retransmissions for radio link error recovery in HSDPA, originate from the base station. Since both functionalities necessitate buffering of data at the radio link interface, 3GPP HSDPA standards provides mechanisms for allocating separate base station data buffers for each end-user or user equipment (UE) connected to the downlink shared channel. In order to facilitate priority handling of multiple flows belonging to the same user, the allocated data buffers can consist of multiple priority queues according to the 3GPP standards. On the other hand, detailed algorithms for priority handling, traffic control and data transmission scheduling at the radio link interface are left out from the standards as open implementation-specific issues. Solutions that address these functionalities are extremely vital to QoS provisioning, end-to-end traffic performance, and efficient radio link utilization, given the buffering of the flows at the radio link interface where bandwidth constraints are frequently encountered due to the time-varying capacity and error-prone nature of the radio channel. An effective solution is to incorporate buffer management algorithms for intra-user traffic control and priority handling in the base station. Mechanisms to incorporate buffer management algorithms to optimize traffic performance in the radio access network already exist within the current 3GPP HSDPA standards. Hence, in this paper we propose and investigate novel radio link buffer management schemes for multimedia sessions with concurrent RT and NRT classes of flows being transmitted to the same user. The proposed buffer management schemes are based on Time-Space priority queuing- a hybrid queuing strategy that allows preferential treatment to real-time and non-real-time flows according to their QoS requirements. The paper presents a comparative study of the Time-Space priority queuing system with conventional priority queuing schemes by means of stochasticanalytic models. Furthermore, extensive end-to-end performance evaluation studies, using detailed system-level HSDPA simulations to investigate the impact of the proposed Time-Space priority queuing based buffer management algorithms on traffic end-to-end QoS, system performance and resource utilization, are also presented.

\subsection{Related Works}

Buffer management is a fundamental technology to provide QoS control mechanisms, by controlling the assignment of buffer resources among different flows or aggregation of flows according to certain policies [4]. Buffer management schemes can be categorized into three classes from resource management viewpoint, namely: Complete Buffer Partitioning (CBP), Complete 
Buffer Sharing (CBS) and Partial Buffer Sharing (PBS) [5]. From a queuing strategy viewpoint, buffer management can be categorized into time priority and space priority. For finite buffer systems, a queuing strategy is composed of the service (selection) discipline and the buffer access control (BAC) discipline [6]. The former deals with the rule(s) of selecting the next packet(s) for transmission, while the latter is concerned with the rule(s) of accepting new arriving packets. If the priority rule is enforced at the service discipline such that preferential treatment is given to some classes of flows in order to control their end-toend delay and jitter, then the mechanism is classified as time priority. On the other hand, enforcing the priority rule at the buffer access control, such that preferential access to buffer space is given to some classes of flows in order to control their packet loss rate, yields a space priority scheme. In the literature, time priority schemes are also known as delay differentiated schemes while space priority schemes are commonly referred to as loss differentiated schemes. Generally speaking, in priority queuing systems, preferential treatment given to one service/traffic class is always achieved at the expense of the other classes. But, introducing priority queuing mechanisms provide a flexible approach to provision QoS which results in better network resource utilization than dimensioning the network to satisfy the most stringent QoS requirements [7].

Most existing priority queuing and buffer management schemes in the literature are either delay or loss differentiated focussing on either time (service) or space prioritization of the different classes of flows. Several space priority-based schemes have been proposed, many of which have been applied in ATM networks to provide different levels of packet loss rate control for buffered flows with different loss priorities. Two main mechanisms for space priorities are Partial Buffer Sharing (PBS) and push-out (PO). In PBS-based buffer management schemes, packet loss rates of incoming traffic from different (loss) priority classes are controlled based on a threshold in the buffer. When the buffer level is below a pre-determined threshold, PBS accepts both low and high priority packets, but when the buffer level exceeds the threshold, low priority packets cannot access the buffer. High priority packets continue to access the buffer until it is full, at which point arriving high priority packets will also be discarded despite the presence of low priority packets in the buffer. Classical PBS schemes have a static threshold and have been studied in [6], [8-15], for example. In order to improve the overall buffer utilization and responsiveness to changing traffic condition, dynamic threshold PBS schemes have been proposed (see e.g. [4],[16],[17], and [18]). In [19], Awan and Al-Begain, investigate an enhanced PBS scheme where two classes of flows, one of which is delay sensitive (high priority) and the other, loss sensitive (low priority), arrive at the finite buffer. In their proposed PBS scheme, the high priority (delay sensitive) packets are only allowed access to the buffer when the total number of packets is below the pre-determined threshold; otherwise, only the low priority (loss sensitive) packets can access the buffer until it becomes full. While the enhanced PBS scheme in [19], unlike the other loss-differentiated only PBS, provides both delay and loss differentiation, it lacks time/transmission or service prioritization. Despite their packet loss rate control capabilities, the main drawback of employing PBS-based buffer management schemes (or other space priority schemes) in QoS control of concurrent RT and NRT flows, is that the lack of time/transmission prioritization is seriously detrimental to RT flow QoS provisioning.

Complete Buffer Partitioning (CBP) [5] is a buffer resource management policy that segments a given buffer space into multiple queues, according to the differentiated classes of traffic, each of which corresponds to a single class. Traffic belonging to one class cannot occupy the buffer space assigned to another class, resulting in discarded arriving packets even when the overall allocated buffer is not yet full. A CBP scheme with first come first serve (FCFS) service discipline is investigated in [20] for two classes of flows with bursty traffic. In contrast, the Complete Buffer Sharing (CBS) scheme admits packets of any class into a shared allocated buffer as long as there is available space. Thus, an arriving packet of any class is dropped when the buffer is full. Unlike CBP, CBS can achieve high buffer utilization because the entire buffer space is always occupied unless there is shortage of arriving packets. CBS is basically a first-in-first-out (FIFO) queuing discipline with drop-tail packet discarding when an arriving packet encounters a full buffer; it therefore suffers from lack of service differentiation and fairness control. Pushout (PO) schemes are generally considered to be space priority schemes that enhance CBS to support multiple classes (i.e. service differentiation). PO selectively discards the lowest priority packet to allow an incoming higher priority packet to enter the buffer. The main 
drawback of PO schemes is computational complexity, since the lower priority packet has to be 'found' before being overwritten by the high priority packet. Choudhary and Hahne proposed a selective pushout scheme for ATM switches in [7]. The scheme allows an arriving cell to overwrite a cell whose priority is less than or equal to itself from the longest output queue in the buffer when full. Buffer space is completely utilized at high traffic load, with very low overall cell loss rate, as well as very low cell loss rate for the high priority flow. Although PO-based schemes offer improved buffer utilization and overall loss rate performance, they (like other space priority/loss differentiated schemes) are not designed to guarantee delay requirements of RT traffic; and, hence, cannot adequately provide optimum joint QoS control of RT and NRT classes of flows simultaneously being transmitted in an end-user session.

In the state-of-the-art HSDPA cellular network, due to packet buffering at the base station, downlink packet scheduling functionality is one of the main mechanisms employed to enable QoS provisioning. The 3GPP HSDPA specifications allow for separate Node B data buffers for each user connection with priority queuing, thus enabling the design and implementation of different choices of traffic prioritization schemes (i.e. scheduling, buffer management algorithms) to optimize QoS provisioning. Consequently, different packet scheduling algorithms have been proposed for UMTS/HSDPA in several papers amongst which the Proportional Fair [21], [22], [23], Maximum C/I, Round Robin, and variations of these are frequently cited. Majority of these packet scheduling algorithms are designed for inter-user packet scheduling under assumption of single traffic flow type per user. A few proposals such as in [3] and [24] for instance, presented inter-user packet scheduling schemes that allow inter-class prioritization but do not address buffer management for the traffic classes belonging to the same user.

While the proposed scheduling solutions in [3] and [24] provide class differentiation to support users that are running several applications simultaneously, the buffer or queue management aspect is not taken into account. Whereas, because the packet scheduling functionality necessitates data buffering at the edge of the radio access network, where the bottleneck to end-to-end communication exists, buffer/queue management is vital to enhancing network and radio resource utilization as well as improving traffic performance. Most importantly, employing efficient buffer management schemes in the radio access networks will allow for QoS control to cater for the diverse performance requirements of multiple flows simultaneously transmitted to the same user on HSDPA shared channel. Our radio link buffer management approach decouples the inter-class priority handling and control amongst the user's flows, from the inter-user packet scheduling functionality by designing buffer management schemes for the former while re-using existing (inter-user) packet scheduling schemes for the latter. This allows for a flexible schedulerindependent solution which is advantageous since operators are free to choose whichever (inter-user) scheduling scheme suits their requirements. In this paper, we focus on the design and performance evaluation of buffer management schemes for diverse mixed (real-time and non-real-time class) traffic in an end-user session which can be coupled with existing (inter-user) packet scheduling schemes to provide end-to-end QoS provisioning.

\subsection{Paper Contribution}

In this paper, two new HSDPA radio-link buffer management schemes for QoS control of multimedia traffic with concurrent RT and NRT flows transmitted to the same user, are presented and investigated. The first one is the Enhanced Time Space Priority buffer management scheme (E-TSP), which incorporates Time-Space priority queuing mechanism with a credit-based flow control algorithm to mitigate buffer overflow. The E-TSP credit-based flow control algorithm is designed to take into account in its operation, the user terminal's radio link quality, the base station buffer occupancy, and the arrival rates of the user's incoming RT and NRT flows. While E-TSP provides an effective solution for joint intra-user RT and NRT QoS control in the HSDPA radio-link buffers, in certain multi-class traffic mix where persistent, high bit-rate RT flow arrival is present, the NRT flow may experience periods of radio link bandwidth starvation. Hence, a second radio link buffer management scheme is proposed to alleviate potential NRT bandwidth starvation problem. The second scheme is termed the Dynamic Time Space Priority buffer management (D-TSP). D-TSP incorporates a dynamic time/transmission priority switching between the RT and NRT flows. 
While E-TSP and D-TSP are proposed as radio link buffer management schemes for end-to-end QoS enhancement of end-user multi-class traffic in HSDPA, the concepts of the schemes are equally applicable to similar fixed wireless or mobile systems with a common downlink channel shared by several users.

E-TSP and D-TSP are based on our novel Time-Space Priority (TSP) queuing system which, unlike conventional priority queuing systems that have either time prioritization (delay differentiated) or space prioritization (loss differentiated), offers both delay and loss differentiation in a combined time- and space-priority queuing scheme; thus making TSP suitable for joint RT (delay sensitive) and NRT (loss sensitive) QoS control and optimization. In TSP queuing, RT flow is given time priority by default but is only allowed access to part of the allocated buffer, while NRT flow enjoys space priority by unrestricted access to end-user allocated buffer space. In this paper, we have developed analytical models for comparative analyses of TSP with conventional priority queuing disciplines; and we discuss their relative merits and demerits for concurrent RT and NRT QoS control, through performance measures such as mean delay and packet loss probability. Furthermore, end-to-end performance evaluation of the proposed TSP-based buffer management schemes using extensive system-level HSDPA simulations of example multimedia communication scenarios, are presented to demonstrate the effectiveness and design trade-offs of the proposed solutions.

The rest of the paper is organised as follows. The next section gives a background description of the 3GPP HSDPA system in order to put our proposed solutions into context. Next, TSP queuing mechanism is described, together with the development of analytical models for comparative study with conventional queuing disciplines. Details of our new buffer management schemes, E-TSP and D-TSP, are presented next, followed by description of the HSDPA system simulation set up for their end-to-end performance evaluation. Next, the numerical results of end-to-end E-TSP and D-TSP simulation studies are presented and discussed. Finally, we present the conclusions of the findings in this paper and highlight possible areas for future work.

\section{HSDPA SYSTEM DESCRIPTION}

\subsection{General Overview}

HSDPA utilizes a shared channel (HS-DSCH) to transmit data to the User Equipments (UE) over the downlink of a HSDPAenhanced UMTS cell. Compared to Release 99 UMTS, shorter connection and response times and higher cell throughput can be achieved with HSDPA. An overview of HSDPA network topology is shown in Figure 1. It consists of three interacting domains; Core Network (CN), UMTS Terrestrial Radio Access Network (UTRAN) and the UE (User Equipment). The CN is responsible for switching, transit and routing of user traffic. UTRAN provides the air interface access for the receiver and handles all radio related functionalities. UTRAN consists of a Radio Network Controller (RNC) and base station or Node B. The Radio Network Controller (RNC) is the network element responsible for the control of the radio resources of the Node Bs connected to it, and also serves as the service access point that the UTRAN provides to the CN. The main elements of the CN are adopted from GSM, i.e. Serving GPRS Support Node (SGSN) and the Gateway GPRS Support Node (GGSN), through which the HSDPA network connects to external packet switched networks.

The main changes in UMTS (Release 99) to introduce HSDPA (Release 5), have been made to the Node B entity [25]. In Release 99 UMTS, Node B performs air interface processing which includes channel coding and interleaving, rate adaptation, spreading etc., and some basic Radio Resource Management operations such as inner loop power control. Whereas, in HSDPA, the Node B has been equipped with a new MAC layer, called the MAC-hs, which has extended its functionality to include Adaptive Modulation and Coding (AMC), Hybrid-ARQ, and fast Packet Scheduling. In addition, transmission time interval (TTI) is reduced to $2 \mathrm{~ms}$ to enable fast tracking of varying channel conditions required with the new functionalities. 




Figure 1: HSDPA Network topology overview.

\subsubsection{Adaptive Modulation and Coding}

HSDPA allows higher order 16 QAM modulation in addition to QPSK. A given combination of modulation scheme and allowed coding rate comprises a modulation and coding scheme (MSC). The basic principle of Adaptive Modulation and Coding (AMC) is to change the modulation and coding scheme for data transmission in accordance with the variations in channel conditions of the UE. The basic unit of data transmission is known as a Transport Block whose size varies with the selected AMC scheme, such that when receiver has good channel conditions, AMC allows for larger Transport Block. Channel conditions estimated by UE are fed back to Node B using Channel Quality Indicator (CQI) via the uplink control channel.

\subsubsection{Hybrid Automatic Repeat reQuest (HARQ)}

The HARQ entity in HSDPA is responsible for retransmission of data that the UE is unable to decode due to radio transmission errors. HARQ operates in the physical layer of the Node B allowing for fast re-transmission of erroneous packets. Two kinds of HARQ schemes can be used in HSDPA: Chase Combining and Incremental Redundancy [1]. HARQ operation is handled by several HARQ processes which operate independently to yield an $\mathrm{N}$-channel stop-and-wait operation, where $\mathrm{N}$ is the number of processes. Only one HARQ process is allowed per TTI for a given UE transmission.

\subsubsection{Packet Scheduling}

In Release 99 UMTS, the RNC is responsible for packet scheduling over a minimum TTI of 10ms. In HSDPA, the packet scheduling functionality is performed in the Node B with a shorter TTI of $2 \mathrm{~ms}$. The basic function of a HSDPA packet scheduler is to determine which user will receive transmission in the next TTI. The size of the data block transmitted in the TTI frame is determined by the AMC scheme selected, which in turn is based on the channel quality reported via the feedback uplink control channel. In addition to the UE channel quality information, ACK/NACK feedback is also carried on the return uplink channel for the HARQ operation.

In the 3GPP standards, packet scheduling is specified as a MAC-hs functionality as shown in Figure 2. Additionally, priority handling functionality is defined, because multiple queues can exist for a single user with separate flows being transmitted to the UE. However, the standards do not provide implementation details. Our proposed buffer management schemes are designed to fulfil not only the priority handling in MAC-hs but also to enable QoS control for improved end-to-end traffic performance. 


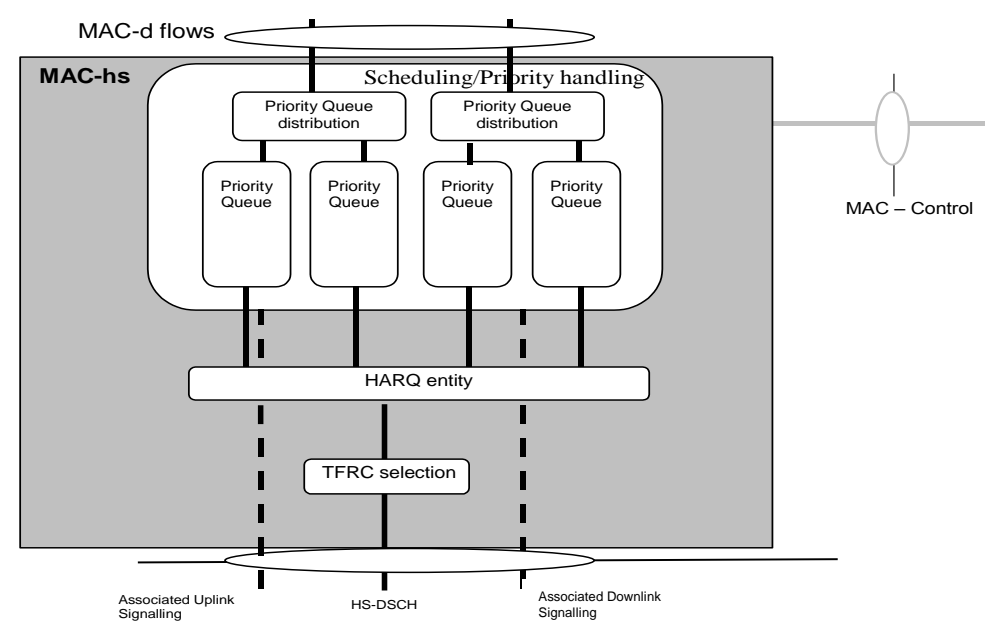

Figure 2 : UTRAN side (Node B) MAC-hs architecture in HSDPA standard [26].

\subsection{Data Flow Mechanisms in HSDPA}

In this section, the main protocol entities and mechanisms involved in a typical HSDPA data flow are described. Referring to the user plane protocol architecture shown in Figure 3, data packets from an external network are routed to the required RNC, by the GGSN via the SGSN after all the connection establishment procedures are completed. In the RNC, the Packet Data Convergence Protocol (PDCP) carries out header compression if required, to improve efficiency of packet switched data transmission over the UTRAN and radio interface. The packets are then segmented/concatenated to generate RLC MAC Protocol Data Units (MAC-d PDUs) for onward transmission to the Node B. Depending on the type of service, RLC MAC-d PDUs can be transferred in either the Acknowledged Mode (AM) or the Unacknowledged Mode (UM) in HSDPA.

The RLC protocol is active in both the RNC and the UE. For PDUs transferred using AM, an ARQ (Automatic Repeat request) retransmission protocol is used for error recovery. The AM is the RLC mode used for TCP-based packet switched services such as web browsing, file downloads, and email for guaranteed data delivery. Segmentation, concatenation, padding and duplicate detection are provided by adding header fields to the data when generating the RLC PDUs from the higher layer packets. When error/loss recovery fails in the RLC AM entity, the application relies on TCP retransmission to recover the lost packets.

In the UM mode, no retransmission protocol is used, which means data delivery is not guaranteed. An RLC entity in the UM mode is defined as unidirectional because no association is needed between the uplink and the downlink. UM is used with applications transported using UDP transport protocol such as VoIP or Streaming audio/video in which RLC level retransmissions are not required or useful. The integrity of higher layer data can be observed using the sequence number in the PDU header which are added to the data to provide segmentation, concatenation, and padding. The complete specification of the HSDPA RLC protocol is given in [27]. 


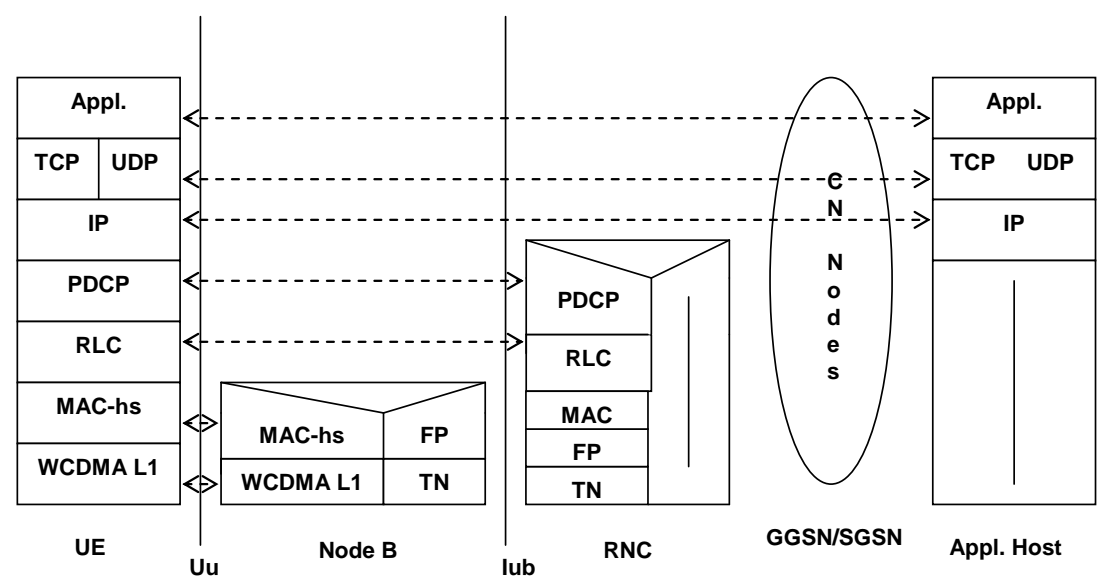

Figure 3: HSDPA User plane protocol stack.

The transfer of AM or UM RLC PDUs to the Node B occurs over the Iub interface using the HSDSCH Frame Protocol (FP). In [28], 3GPP specifies a credit-based Iub flow control mechanism for the transfer of data from the RNC to the Node B using the HS-DSCH FP. Again, the credit-based algorithm employed in practice is an open issue, but the flow control mechanism is aimed at controlling the amount of user data buffered in the MAC-hs of the Node B ready for scheduling on the HS-DSCH to prevent buffer overflow. In the Iub flows control, the Node B issues credits to the RNC through the CAPACITY ALLOCATION FP control frame on receipt of a CAPACITY REQUEST FP control frame from the RNC. The number of credits issued corresponds to the number of RLC PDUs belonging to a particular flow of a given user to be transferred; thus, the RNC packs the PDUs into 10ms HS-DSCH frames to be transmitted to the Node B. The buffer management schemes proposed in this paper are designed to exploit these mechanisms in their incorporated credit-based flow control algorithm.

On arrival of the HS-DSCH frames carrying the RLC PDUs to the Node B MAC-hs, the RLC PDUs are retrieved and queued in the MAC-hs buffer to await transmission. When the packet scheduler assigns a transmission slot to the UE, one or several MAC-d PDUs from its queue(s) are assembled into a data block. A MAC-hs header containing the queue identity, and transmission sequence number etc., is added to form a MAC-hs PDU or Transport Block which is then forwarded to the physical layer for further processing. Recall that the Transport Block Size and hence number of MAC-d PDUs transmitted will depend on the selected AMC schemes and the number of channelization codes which will be chosen according to the Channel Quality Indicator which expresses the last known UE channel conditions. The maximum number of channelization codes allowed for HS-DSCH transmission is 15 , but 5 or 10 codes could be used depending on the terminal capability [29]. Although HS-DSCH spreading factor of 16 allows for up to 16 codes, one code is always reserved for the associated High Speed Shared Control Channel (HS$\mathrm{SCCH}$ ) that carries channel decoding information. A HARQ process is assigned for the transmission of a Transport block to the UE in the allocated TTI, and the Transmission Sequence Number (TSN) in the frame is used to preserve the integrity and the order of the data.

The UE has peer HARQ entities which receives the data frame and sends an ACK if decoding was successful or a NACK otherwise. A reverse feedback channel, the HS-DPCCH is used to carry the HARQ ACK/NACK as well as the Channel Quality Indicator that controls the AMC process. Correctly received frames are de-assembled into MAC-hs PDUs and reordered in the MAC-hs reordering queues. The MAC-d PDUs are extracted and sent to the RLC layer for further processing. A peer RLC AM entity will send a STATUS PDU in the return direction if a POLL bit has been set in the header of the received PDUs. The packets are reconstructed from the RLC PDUs and passed to the higher layer transport protocol for further processing and onward transfer to the application layer. All the above described mechanisms are either explicitly or implicitly modelled in our system-level HSDPA simulator used to evaluate the end-to-end performance of our proposed E-TSP and D-TSP buffer management schemes described in section 4 . 


\section{ANALYSIS OF PRIOIRTY QUEUING SCHEMES}

In this section, the Time-Space priority (TSP) queuing system is described. The conventional priority queue management types commonly encountered in the literature namely, CBS (complete buffer sharing), PBS (partial buffer sharing), and CBP (complete buffer partitioning) are used as reference for comparative analysis to the TSP queue management. Analytical models are formulated using continuous-time Markov Chains and the effects of different traffic, system and configuration parameters on the performance measures like packet loss probability, and mean delay are studied using the model. The study is intended to give insight into merits and constraints of TSP queuing and to assess its capability for joint RT and NRT QoS control compared to the conventional alternatives.

\subsection{Time-Space Priority Queuing System}

The Time-Space priority (TSP) [30] queuing model is a novel hybrid priority queuing system, proposed for joint QoS control of ongoing end-user multi-class sessions with real-time (RT) and non-real-time (NRT) flows queued at the bottleneck downlink node, with transmission over a shared wireless channel. With TSP, a single queue is utilized as shown in Figure 4, with a threshold R, which controls the admission of RT packets into the queue. Since RT flow is delay sensitive, the queuing of arriving RT packets proceeds in a FIFO manner ahead of the NRT packets. On the other hand, RT flow is to some extent loss tolerant, hence the threshold R, is used to limit the total number of RT packets in the queue and at the same time accord the NRT packets buffer space priority. Consequently, RT delay is minimized with time priority, while at the same time, NRT loss is minimized with the space priority mechanism. Note that with TSP queuing, the entire allocated buffer space is completely shared by RT and NRT packets in spite of the threshold R. Hence, the total number of NRT packets allowed in the buffer can range from N, the total queue capacity, to N-R, which further minimizes loss of NRT packets whilst also ensuring a high degree of buffer utilization. NRT packet loss minimization at the radio link interface is extremely vital, since lost NRT packets typically must be retransmitted using (higher layer) error control protocols, which consequently results in end-to-end throughput degradation and inefficient network resource utilization.

The effect of time priorities is to minimize the delay of the RT traffic at the expense of the delay of the NRT traffic, and conversely, the effect of the space priorities is to minimize the loss of NRT packets at the expense of some loss of RT packets. The diverse QoS characteristics of RT (i.e. loss tolerance and delay sensitivity), and NRT (i.e. delay tolerance and loss sensitivity), makes TSP queuing an effective mechanism for optimum QoS control and optimization using the time-space priority threshold. Unlike the existing priority queuing types mentioned in section 1.1, which are designed for either delay or loss differentiation, the uniqueness of TSP lies in its capability to provide both delay and loss differentiation. The TSP queuing system is at the core of the HSDPA buffer management schemes, E-TSP and D-TSP, proposed in this paper. Hence, we provide a preliminary study of TSP queuing by comparison with traditional queuing schemes, before undertaking a performance evaluation of the TSP-based buffer management schemes (i.e. E-TSP and D-TSP).

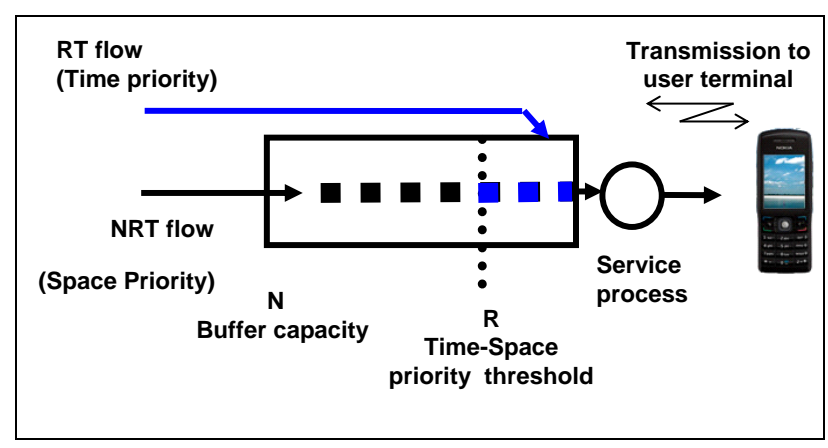

Figure 4: The Time-Space Priority queuing system. 


\subsection{Conventional Priority Queuing Schemes}

Recall from section 1.1 that most existing priority queuing schemes can be classed in to CBS, PBS or CBP, from buffer resource management viewpoint; while in terms of service differentiation strategy priority queuing can be based on time priorities or space priorities. Thus, in order to assess the capability of TSP to enable joint RT and NRT QoS control in a multi-class connection, we compare its performance to the following conventional queuing schemes:

1) PBS (Partial Buffer Sharing): Assuming two levels of priority -high and low- corresponding to NRT and RT classes respectively, PBS operates a selective discard mechanism aimed at minimizing the loss of packets of the high priority flow with respect to the lower priority flow packets. PBS uses a threshold to reserve a part of the buffer for high priority packets only. If the queue size is below the threshold, both high and low priority packets are accepted into the queue. Above the threshold, only high priority (NRT) packets are accepted. Since PBS is the most widely studied space priority scheme, it is taken as a baseline space priority scheme for comparative study.

2) CBP (Complete Buffer Partitioning): Separate buffer partitions are maintained for the RT and NRT flows, without any common buffer sharing. Static time prioritization is applied to always transmit RT packets first before the transmission of NRT packets. CBP is well known for its fairness properties (in buffer space allocation) especially under asymmetric traffic conditions. The CBP scheme is taken as the baseline time-priority scheme for the comparative analyses.

3) CBS (Complete Buffer Sharing): CBS does not utilize thresholds to partition the waiting area of the buffer. Arriving RT and NRT packets are accepted into the queue in a first-come-first-serve manner as long as there is available buffer space while droptail discarding is used when the buffer is full. Even though no delay or loss differentiation is employed, CBS queuing discipline ensures the highest possible buffer utilization when applied to multi-class traffic. Hence, CBS provides a baseline to assess the buffer utilization capability of TSP for multi-class traffic.

\subsection{Formulation of the Analytical Models}

Figure 5 shows the arrangement of the assumed system configuration for the Markov models used in the comparative performance evaluation of the queuing schemes. The system comprises the radio controller (RNC) queues and the base station (BS) queue(s). The policy for admitting packets from the RNC queues into the BS queue(s) and the order of packet transmission, proceed according to the descriptions of the priority queuing schemes given in section 3.2 and set the conditions governing the state transitions in the Markov model.

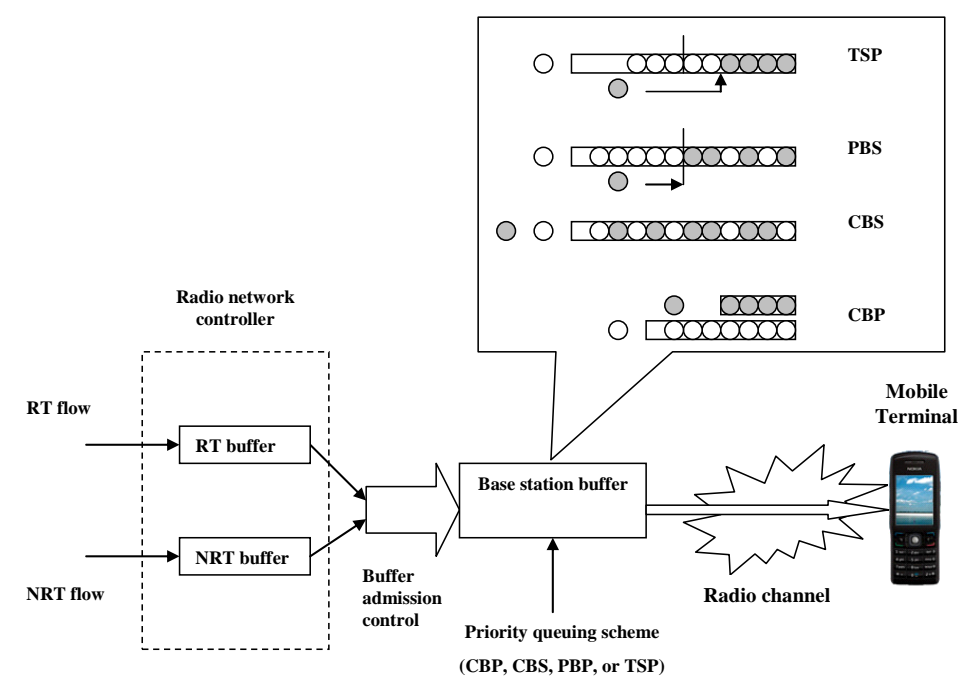

Figure 5: Simplified model for investigating performance of the priority queuing mechanisms for mobile radio access network downlink end-user multi-class traffic. 


\subsubsection{Basic system assumptions and notations:}

- Two classes of traffic, RT and NRT exist within a connection or session with arrival to separate RNC queues according to independent Poisson process with arrival rates $\lambda_{\text {rt }}$ and $\lambda_{\text {nrt }}$ respectively. The RNC queues are of limited capacity hence arriving packets that encounter a full queue are discarded (as shown in Figure 5).

- We assume that packets are transmitted between the RNC and BS buffers without any transmission loss or delay and proceed only when BS buffer admission control policy, which differs with applied BS queuing scheme, permits.

- Let the radio channel state ch assume either a 'good' state or 'bad' state corresponding to favourable and unfavourable channel conditions respectively. Assuming the time spent in each state is exponentially distributed, $c h$ transits from 'good' to 'bad' $(c h=1$ to $c h=0)$ at an average rate of $1 /\left(1-\mathrm{P}_{\mathrm{g}}\right)$ and from 'bad' to 'good' $(c h=0$ to $c h=1)$ on average at the rate of $1 /\left(1-\mathrm{P}_{\mathrm{b}}\right)=1 / \mathrm{P}_{\mathrm{g}}$. Where $\mathrm{P}_{\mathrm{g}}$ denotes probability that $c h$ is in 'good' state and $\mathrm{P}_{\mathrm{b}}=1-\mathrm{P}_{\mathrm{g}}$ denotes the probability of $c h$ remaining in 'bad' state. State $c h$, allows modelling an error-prone radio channel in the Markov chain to capture the effect of packet loss and retransmission on the air interface due to unfavourable radio channel conditions. Thus, packet transmission proceeds on the radio link, only when the channel state $c h$ is 'good'.

- Packet transmission times at the radio interface are assumed to be independent and exponentially distributed with mean $1 / \mu$, with the order of packet transmission depending on the applied BS queuing scheme.

- Let R represent the total RNC RT queue capacity while N represents the total RNC NRT queue capacity. Denote $r$ as the number of RT packets in the RNC RT queue, and $n$ as the number of NRT packets in the NRT RNC queue at any given time.

- Let T represent the total BS queue capacity. Also denote $t=r_{b}+n_{b}$ as the total number of packets present in the base station at any given time, where $r_{b}$ and $n_{b}$ are the number of RT and NRT packets present respectively.

- Denote $R_{b}$ as the TSP threshold in the BS queue i.e. maximum number of allowable RT packets according to TSP policy. Thus, with TSP queuing, RT packets are moved from RNC RT queue to BS only when $r_{b}<R_{b}$, while NRT packets move from RNC NRT queue to BS whenever $\mathrm{t}<\mathrm{T}$.

- Denote $R_{c}$ as the CBP partition threshold which divides the BS queue such that the maximum allowable number of RT packets in the BS queue is given by $R_{c}$ for CBP.

- Let th denote the PBS threshold in the BS queue, when PBS queuing is applied.

- Finally, denote by $p=1$ the state when the head-of-the-line BS queue packet waiting for transmission is an RT packet and $p=0$ otherwise; and by $q=1$, when the head-of-the-line packet awaiting transmission is an NRT packet and $q=0$ otherwise. Empty state of the BS queue is given by $p=0, q=0$.

\subsubsection{The Markov models}

We define the state of the system by a vector $\mathrm{X}=\mathrm{J}^{\prime}$ where $\mathrm{J}^{\prime}=(r, n, t, p, q, c h)$ when TSP or CBP is applied in the BS queue, and $\mathrm{J}^{\prime}=(r, n, t, c h)$ when either PBS or CBS policy is applied in the BS queue. Let the number of possible states in the Markov chain be given by $M$. We can thus define $K$ to represent the finite state space of the system given that the states are conveniently ordered from $0 \ldots M-1$. Since X comprises several elements defining the system state space with each of the applied queuing schemes, a multi-dimensional Markov model is formulated to capture the applied scheme's mechanism in the system with defined states and transitions from which steady state probabilities and hence performance measures can be computed. If we denote by $\mathbf{Q}$, the generator matrix of each of the multi-dimensional Markov models, which are homogeneous and irreducible on the finite state space $K$, a unique steady state distribution $\quad \mathbf{P}=\left\{\mathrm{P}_{\mathrm{x}}\right\}, \mathrm{x}=0 \ldots \mathrm{M}-1$, exists.

The solution of the matrix equation $\mathbf{P} . \mathbf{Q}=\mathbf{0}$, subject to $\sum_{x=0}^{M-1} \mathrm{P}_{\mathrm{x}}=1$, yields the steady state probabilities $\mathrm{P}_{\mathrm{x}}, \mathrm{x}=0 \ldots \mathrm{M}-1$, from which the performance measures of interest are calculated. The elements of the matrix $\mathrm{Q}$ are given by the transition rates from a state $\mathrm{X}=\mathrm{J}^{\prime}$ to a succeeding state $\mathrm{X}^{+}=\mathrm{J}^{\text {'+ }}$ or a preceding state $\mathrm{X}^{-}=\mathrm{J}^{\text {'- }}$, where $\mathrm{J}^{\text {'+ }}$ denotes a discrete increment in a vector element 
by one and $\mathrm{J}^{\prime}$ - denotes a decrement in a vector element by one, given an initial state $\mathrm{X}^{0}=\mathrm{J}^{\prime 0}$. The transitions and rates are governed by conditions which are defined by the chosen BS queuing mechanism under the assumptions described in section 3.3.1. Since $c h$ has two possible states $(0$ and 1$)$ and $(p, q)$ have three possibilities of allowed combined states $((0,0),(0,1),(1,0))$, the sizes of the finite state space required to compute the performance measures from the multi-dimensional Markov model are given by:

$S=(R+1) *(N+1) *\left(T-R_{c}+1\right) *\left(R_{c}+1\right) * 2 * 3$ for CBP,

$S=(R+1) *(N+1) *(T+1) * 2$ for PBS or CBS,

$S=2 * 3 *(R+1) *(N+1) *\left(\left(\left(R_{b}+1\right)(T+1)\right)-\left(\frac{R_{b}\left(R_{b}+1\right)}{2}\right)\right)$ for TSP.

Thus, $\mathbf{Q}$ will be an $\mathrm{S} * \mathrm{~S}$ square matrix while $\mathbf{P}$ is a vector of dimension $\mathrm{S}$. Clearly, even for small $\mathrm{R}$ and $\mathrm{N}$ and $\mathrm{T}$ values, the size of the state space and hence the recursive linear equations required for the solution of the steady state probability matrix equation can be quite large. Hence, we apply the Markov modelling tool, MOSEL-2 [31-33] to generate the state space and numerically solve the Markov model using Stochastic Petri Net Package (SPNP) [34] package to obtain the performance measures of interest defined as follows:

- RT packet loss probability:

$L_{r t}=\sum_{x \in k} P(x)$

For TSP: $k=\left\{\mathrm{x}: r=R, n \in\{0,1, \ldots, N\}, r_{b}=R b, p, q \in\{(0,0),(1,0),(0,1)\}, c h \in\{0,1\}\right\}$

For CPB: $k=\left\{\mathrm{x}: r=R, n \in\{0,1, \ldots, N\}, r_{b}=R c, p, q \in\{(0,0),(1,0),(0,1)\}, c h \in\{0,1\}\right\}$

For PBS: $k=\{\mathrm{x}: r=R, n \in\{0,1, \ldots, N\}, t \geq t h, \operatorname{ch} \in\{0,1\}\}$

For CBS $k=\{\mathrm{x}: r=R, n \in\{0,1, \ldots, N\}, t=T, \operatorname{ch} \in\{0,1\}\}$

which define the set of all the states of the system in which an arriving RT packet to the RNC RT buffer will be discarded for the various schemes.

- NRT packet loss probability:

$L_{n r t}=\sum_{x \in k} P(x)$

For TSP: $k=\{\mathrm{x}: r \in\{0,1, \ldots, R\}, n=N, t=T, p, q \in\{(0,0),(0,1),(1,0)\}, c h \in\{0,1\}\}$

For CBP: $k=\{\mathrm{x}: r \in\{0,1, \ldots, R\}, n=N, t=T-R c, p, q \in\{(0,0),(1,0),(0,1)\}, \operatorname{ch} \in\{0,1\}\}$

For PBS and CBS: $k=\{\mathrm{x}: r \in\{0,1 \ldots, R\}, n=N, t=T, c h \in\{0,1\}\}$

which all define the set of all possible states of the system in which an arriving NRT packet to the RNC NRT buffer will be discarded.

- In all schemes, the mean queue length of the RNC RT buffer is given by:

$N_{r t}=\sum_{x \in k} r P(x)$ where $r \in\{0,1, \ldots, R\}$ in all possible states $k$.

- In all schemes, the mean queue length of the RNC NRT buffer is given by:

$N_{n r t}=\sum_{x \in k} n P(x)$ where $n \in\{0,1, \ldots, N\}$ in all possible states $k$.

- From Little's law, mean delay for RT packets is calculated from [35]:

$D_{r t}=\frac{N_{r t}}{\lambda_{r t} \times\left(1-L_{\mathrm{r} t}\right)}$

- From Little's law, mean delay for NRT packets is calculated from:

$D_{n r t}=\frac{N_{n r t}}{\lambda_{n r t} \times(1-L n r t)}$ 


\subsection{Numerical Results and Discussions}

Numerical results are presented in this section to illustrate the effects on the performance metrics, when each of the queuing schemes are employed in the BS buffer of the system shown in Figure 5, modelled with the continuous-time Markov Chain models formulated above. The default system parameter values assumed in the Markov models used in the experiments are summarized in Table 1. A range of input traffic configurations with different NRT to RT flow traffic load ratios are considered. The total multiplexed traffic load is kept constant, while the RT packet loss probability, NRT packet loss probability, mean RT delay, and total (RT and NRT) packet loss, are taken as performance indicators for comparative analysis.

Table 1: Values of default parameters used in analytical models for comparative performance evaluation of the priority queuing schemes.

\begin{tabular}{|l|c|}
\hline \multicolumn{1}{|c|}{ Parameter } & Value \\
\hline Mean total arrival rate (to RNC queues), $\lambda$ & 10 \\
\hline Mean service rate (at radio interface), $\mu$ & 12 \\
\hline Probability of good channel state, $\mathrm{P}_{\mathrm{g}}$ & 0.8 \\
\hline RNC RT queue capacity, $\mathrm{R}$ & 5 \\
\hline RNC NRT queue capacity, $\mathrm{N}$ & 10 \\
\hline BS total queue capacity, $\mathrm{T}$ & 4 \\
\hline TSP scheme threshold in BS queue, $R_{b}$ & 4 \\
\hline CPB scheme threshold in BS queue, $R_{c}$ & 4 \\
\hline PBS scheme threshold in the BS queue, $t h$ & $0.2,0.4,0.6,0.8$ \\
\hline traffic ratio & $\lambda *$ traffic ratio \\
\hline Mean NRT arrival rate, $\lambda_{\text {nrt }}$ & $\lambda-\lambda_{\text {nrt }}$ \\
\hline Mean RT arrival rate, $\lambda_{\text {rt }}$ & 5 \\
\hline
\end{tabular}

Figure 6 shows the mean RT delay for the compared schemes under NRT to total input traffic ratios of $0.2,0.4,0.6$, and 0.8 respectively. A parameter value of 0.2 , for example, means that on average $20 \%$ of packet arrivals are from the NRT flow while the remaining $80 \%$ are from the RT flow. Conversely, a parameter value of 0.8 indicates that $80 \%$ of packet arrivals are from the NRT flow while the remaining $20 \%$ are from the RT flow. This metric allows us to capture a wide range of multi-class traffic configurations and is used throughout the experiments.

From Figure 6, it can be seen that TSP and CBP generally achieve the lowest mean RT flow delay due to RT service prioritization. With CBS applied in the BS, RT packets can traverse the system more easily than with PBS, because of better fairness in access to BS buffer space that CBS provides for RT flow compared to PBS which utilizes a threshold to limit access to BS buffer space. For this reason, CBS achieves lower mean RT delay compared to PBS. Note that even though TSP also uses a limiting threshold, $R_{b}$, the precedence queuing and service (time) priority for RT flow, which are features absent in PBS, not only minimizes RT queuing delay but also allows transfer of RT packets from RNC to BS if there is available space while $r_{b}<R_{b}$ in the BS. Whereas, with PBS, even when BS buffer space is available as long as $t \geq t$ (th $=R_{b}$ in the experiments), RT packets cannot go through the system. An increase in the traffic ratio on the x-axis represents increase in NRT traffic component and a corresponding decrease in RT component; the effect of this is apparent in the PBS curve behaviour. That is, the increase in the NRT flow component causes further stalling of RT flow in the RNC RT queue, leading to corresponding increase in delay. The opposite effect can be observed (to a lesser extent) in the TSP or CBP curves; i.e. RT delay lowers with decreasing RT flow component. From Figure 7, it can be observed that the lowest RT packet loss probabilities are generally obtained with TSP and 
CBP. PBS gives the highest RT packet loss probabilities. Similar to the case of delay performance in Figure 6 , the observed better TSP RT loss performance is due to service (time) prioritization present in the scheme.

Figure 8 plots the NRT packet loss probabilities for all the schemes. Due to space priority mechanism in PBS for NRT flow, PBS showed the lowest NRT loss probability, followed by CBS, TSP and CBP respectively. Because of higher buffer utilization inherent in TSP queuing compared to CBP, it achieved better NRT loss performance than the latter. Figure 9 shows that overall packet loss is highest with PBS, while overall packet loss is lowest with CBS. TSP gives lower overall packet loss than CBP, indicative of better BS buffer space utilization in TSP compare to CBP. As stated before, the CBS scheme always achieves a high degree of buffer utilization, which is the reason for the lowest overall packet loss when used in the BS compared to the others. Note also that TSP performance comes closest to CBS in Figure 9, illustrating the high buffer utilization capability of TSP as well.

The results provide insight into the comparative behaviours of the schemes over a range of multi-class traffic configurations. The drawback of PBP and CBS compared to TSP is the tendency to compromise RT class QoS. On the other hand, the drawback of CBP is the lower buffer utilization and tendency to achieve lower NRT QoS compared to an equivalent TSP queue. This leads to the conclusion that compared to the typical priority queuing disciplines, TSP is the most effective queuing scheme that can be used achieve a compromise between QoS of RT and NRT classes of flows that are concurrent in a multi-class session, whilst also enabling a high degree of buffer utilization and low overall packet loss.

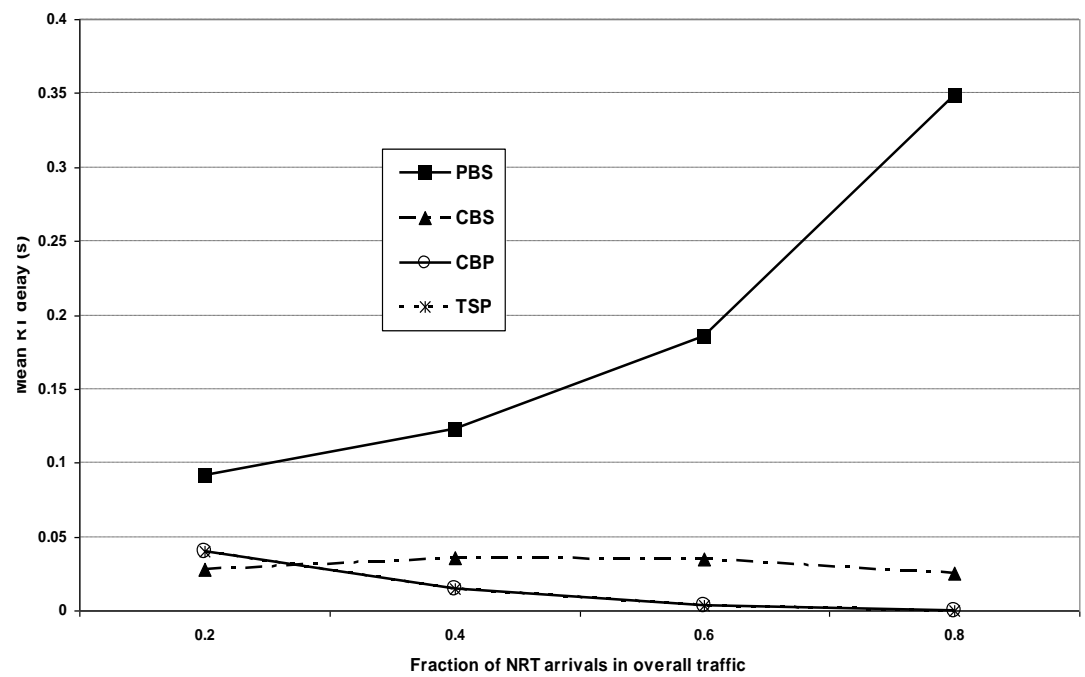

Figure 6: Mean RT delay vs. fraction of NRT arrivals in concurrent RT and NRT traffic for TSP, CBP, PBS, and CBS queuing.

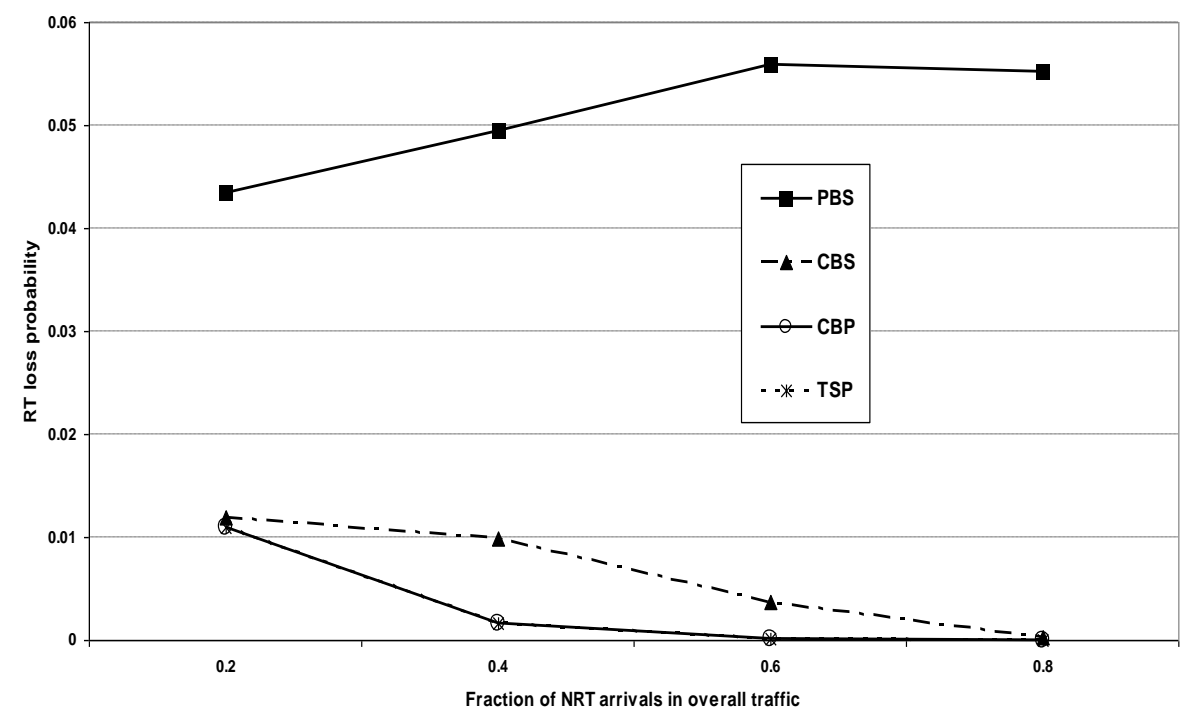

Figure 7: RT packet loss probability vs. fraction of NRT arrivals in concurrent RT and NRT traffic for TSP, CBP, PBS, and CBS queuing. 


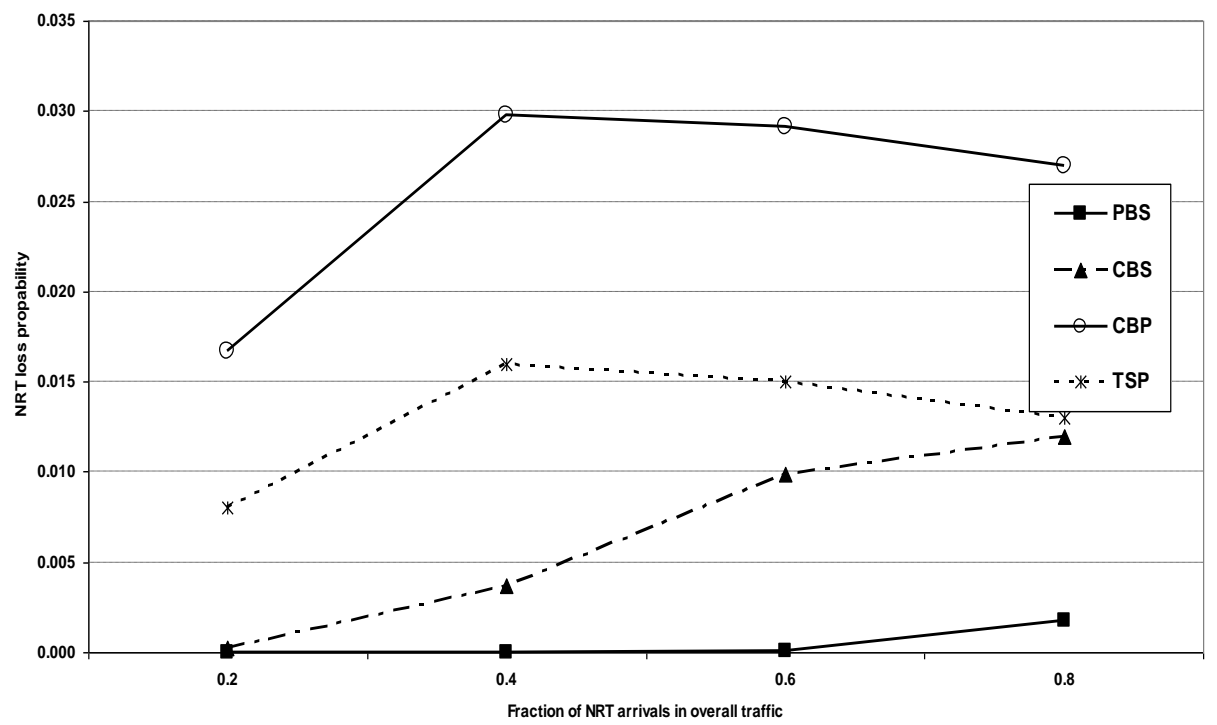

Figure 8: NRT packet loss probability vs. fraction of NRT arrivals in concurrent RT and NRT traffic for TSP, CBP, PBS, and CBS queuing.

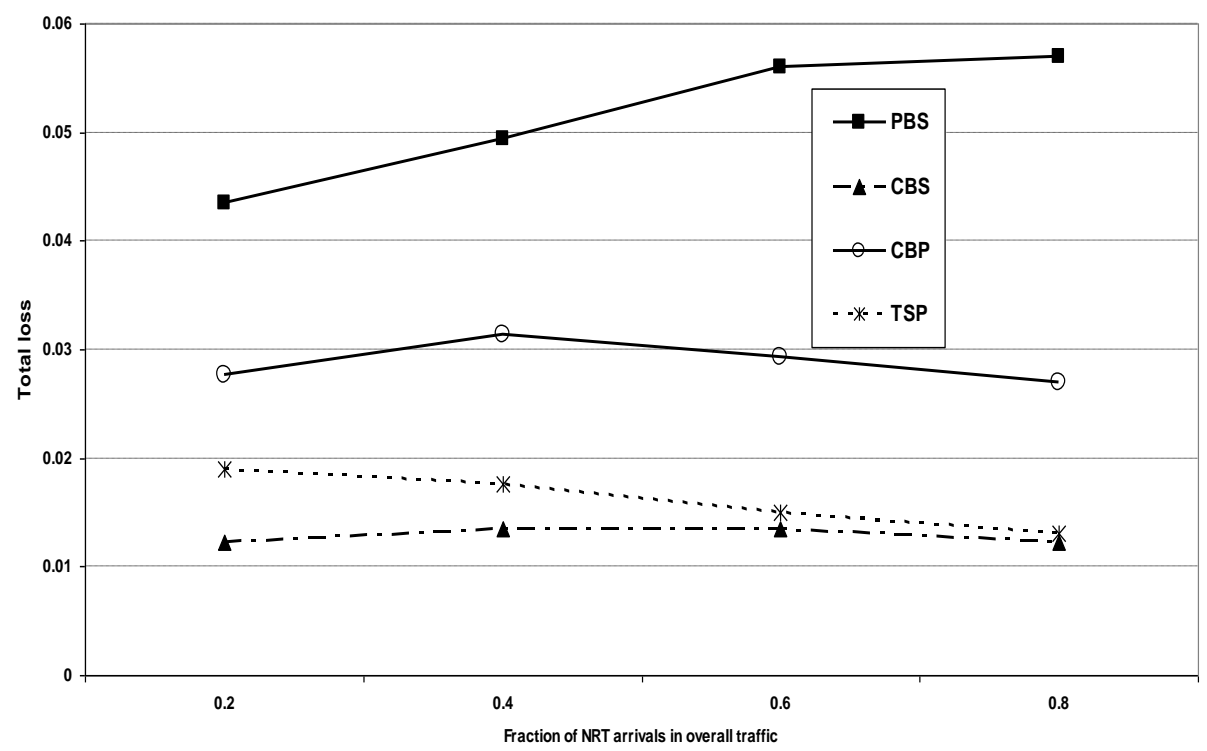

Figure 9: Overall packet loss probability vs. fraction of NRT arrivals in concurrent RT and NRT traffic for TSP, CBP, PBS, and CBS queuing.

\section{PROPOSED HSDPA BUFFER MANAGEMENT SCHEMES}

Having evaluated TSP queuing against conventional schemes, we devote the following sections to description and evaluation of our proposed TSP-based HSDPA buffer management schemes.

\subsection{The Enhanced Time-Space Priority (E-TSP) Buffer Management Scheme}

TSP, despite being more effective than conventional queuing schemes for joint RT and NRT QoS control of end-user multi-class traffic, uses drop-tail queuing. Because of drop-tail queuing when the buffer is full, deploying TSP at the radio link of practical wireless systems like HSDPA, could adversely affect end-to-end QoS where TCP is used to transport one or more of the flows in the multi-class session. It is therefore desirable to design pro-active radio link buffer management schemes for multi-class traffic in order to alleviate the side effects of buffer overflow, which can negatively impact end-to-end throughput. HSDPA standard specifies mechanisms [36] for (credit-based) Node B flow control where credits are sent to the RNC to regulate the flow of traffic 
to the RNC. Hence, we propose an Enhanced Time-Space priority buffer management (E-TSP) for HSDPA multi-class sessions with concurrent RT and NRT flow illustrated in Figure 10.

In addition to TSP queuing, E-TSP incorporates a (credit-based) flow control algorithm necessitating the additional thresholds L and $\mathrm{H}$ besides the TSP threshold, R. Thus the overall E-TSP logical queue incorporates three thresholds as shown in Figure 10. The proposed Iub flow control algorithm uses the HS-DSCH Frame Protocol explained in section 2.2, to issue credits which gives the number of Protocol Data Units (PDUs) allowed to be transmitted from the RNC. Furthermore, our algorithm is designed to react to variation of the UE channel conditions, HSDPA channel load, as well as buffer occupancy which not only mitigates buffer overflow but also achieves more efficient radio link utilization. The basic idea of the E-TSP flow control algorithm is that when buffer occupancy exceeds the lower flow control threshold L, the NRT arrival rate (controlled by credit-allocation) is reduced. If the buffer occupancy exceeds the higher flow control threshold H, no credits are issued i.e. instantaneous NRT arrival is reduced to zero.

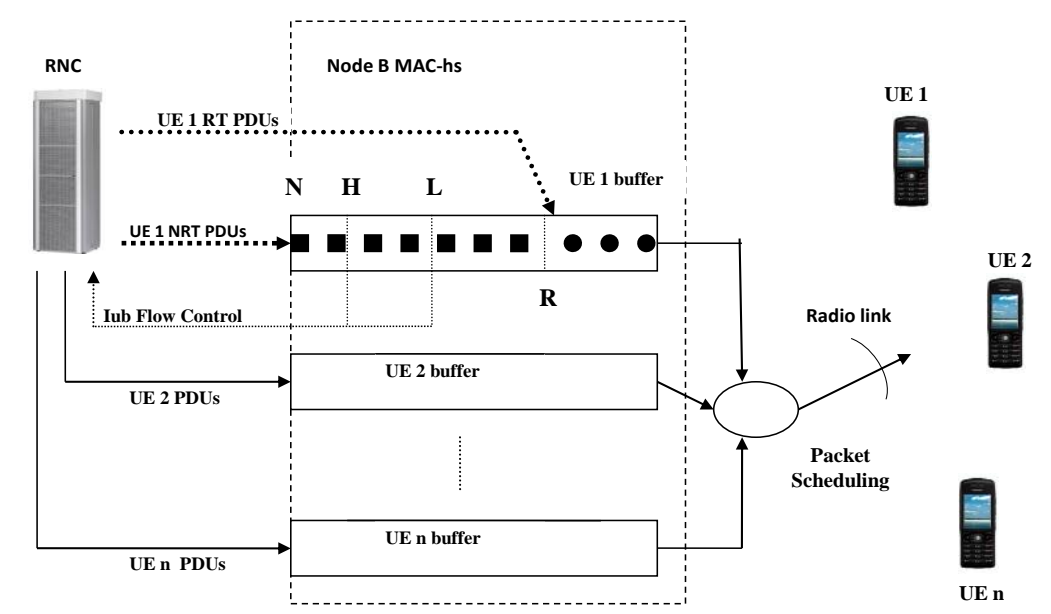

Figure 10: E-TSP buffer management scheme for multi-class traffic UE in Node B.

The E-TSP algorithm is described with the following assumptions and notations:

- Assuming a total buffer allocation of N PDUs for $\mathrm{UE}_{\mathrm{n}}$, in the Node B MAC-hs, where $\mathrm{n}$ is the nth user in the HSDPA cell. Let R denote the total number of allowed RT PDUs in the nth user's MAC-hs buffer.

- Let $r(t)$ be the number of nth user's RT PDUs in the buffer at time $t$, while we denote the number of nth user's NRT PDUs at time $\mathrm{t}$ as $\mathrm{n}(\mathrm{t})$. Thus from TSP principle $0<\mathrm{r}(\mathrm{t})<\mathrm{R}$ and $0<\mathrm{n}(\mathrm{t})<\mathrm{N}$ at any given time $\mathrm{t}$.

- Denote the lower Iub flow control threshold as L, where L > R. Likewise the higher flow control threshold is given by H, where $\mathrm{L}<\mathrm{H}<\mathrm{N}$.

- Let the nth user's buffer occupancy at time $t$ be given by $q(t)=r(t)+n(t)$. The average buffer occupancy is estimated using a moving average filter with ith sample given by:

$$
\mathrm{q}_{\mathrm{i}}=w \cdot \mathrm{q}_{(\mathrm{i}-1)}+(1-w) \cdot \mathrm{q}(\mathrm{t})
$$

- Denote $\lambda_{\mathrm{rt}}$ as the Guaranteed Bit Rate (GBR) of the RT flow (obtainable from bearer negotiation parameters [37]).

- Let $\lambda_{\text {nrt }}$ express the estimated average NRT flow data rate at the radio interface determined from:

$$
\lambda_{\text {nrt (i) }}^{\prime}=\alpha \cdot \lambda_{\text {nrt (i-1) }}^{\prime}+(1-\alpha) \cdot \lambda_{\text {nrt }}(\mathrm{t})
$$

where $\mathrm{i}$ is the ith TTI in which the user's NRT flow was transmitted during a UE scheduling opportunity and $\lambda_{\text {nrt }}(\mathrm{t})$ is the amount of NRT data transmitted during the ith TTI.

- Let $k$ denote a parameter for buffer overflow control, while $\mathrm{T}_{\mathrm{f}}$ and PDU_size be HS_DSCH FP inter-frame period (10ms) and the MAC-d PDU size in bits, respectively. 
Given the above assumptions and notations, E-TSP scheme in HSDPA operates as follows:

\section{Part 1: User n Credit allocation:}

- Step 1: Compute per frame RT flow credit allocation

$$
\mathrm{C}_{\mathrm{rt}}=\left(\lambda_{\mathrm{rt}} / \mathrm{PDU} \_ \text {size }\right) \cdot \mathrm{T}_{\mathrm{f}}
$$

- Step 2: Compute per frame maximum NRT credits

$$
\begin{aligned}
\mathrm{C}_{\text {NRTmax }}=\left(\lambda_{\text {nrt }}^{\prime} / \text { PDU_size }\right) \cdot \mathrm{T}_{\mathrm{f}} & \text { if } \quad \mathrm{q}_{\mathrm{i}}<\mathrm{L} \\
k \cdot\left(\lambda_{\text {nrt }}^{\prime} / \mathrm{PDU} \_ \text {size }\right) \cdot \mathrm{T}_{\mathrm{f}} & \text { if } \mathrm{L} \leq \mathrm{q}_{\mathrm{i}} \leq \mathrm{H} \\
0, & \text { if } \quad \mathrm{q}_{\mathrm{i}}>\mathrm{H}
\end{aligned}
$$

- Step 3: Compute per frame NRT credit allocation

$\mathrm{C}_{\mathrm{NRT}}=\min \left\{\mathrm{C}_{\mathrm{NRTmax}}, \mathrm{RNC}_{\mathrm{NRT}}\right\}$ where $\mathrm{RNC}_{\mathrm{NRT}}$ is the RNC NRT flow buffer occupancy.

- Step 4: Compute total per frame credit for nth user

$\mathrm{C}_{\mathrm{T}}=\mathrm{C}_{\mathrm{rt}}+\mathrm{C}_{\mathrm{NRT}}$

Part 2: TSP queue management:

- Step 1: For each arriving HS-DSCH data frame from RNC for user $n$ determine the flow class - RT or NRT.

- Step 2: If flow belongs to RT class, for each MAC-d PDU in the payload:

If $(\mathrm{r}(\mathrm{t})<\mathrm{R})$ qиеие PDU at RT quеие tail

Else drop MAC-d PDU and update RT loss

Else If flow belongs to the NRT class, for each MAC-d PDU in the payload:

If $\mathrm{r}(\mathrm{t})+\mathrm{n}(\mathrm{t})<\mathrm{N}$ quеие PDU at buffer queие tail

Else drop MAC-d PDU and update NRT loss

\section{Part 3: Transmission priority control:}

- For each $\mathrm{UE}_{\mathrm{n}}$ transmission opportunity:

IF $(r(t)>0)$

\section{\{}

Time Priority $=$ RT flow

Generate MAC-hs Transport Block from RT PDUs

$$
\}
$$

$\operatorname{ELSE~IF~}(\mathrm{r}(\mathrm{t})==0$ AND $\mathrm{n}(\mathrm{t})>0)$

\{

Time Priority $=$ NRT flow

Generate MAC-hs Transport Block from NRT PDUs

\}

Note that NRT grant allocation and hence NRT flow arrival rate is made dependent on channel load and UE channel quality through equations (2) and (4). Generally, the better the channel quality or the lighter the cell load, the larger $\lambda$ ' ${ }_{\text {nrt }}$ estimate hence the more credits get allocated and vice versa. Since averages are used in the grant calculation, and because of possible time-lag between issued credit and $\mathrm{RNC}$ reaction, the space between $\mathrm{H}$ and $\mathrm{N}$ absorbs instantaneous burst arrivals that could occur even whilst $\mathrm{C}_{\mathrm{NRTmax}}=0$.

\subsection{The Dynamic Time-Space Priority (D-TSP) Buffer Management Scheme}

While E-TSP algorithm (designed to address the potential buffer overflow, reduce excessive radio link buffer queuing delays, and for better radio link utilization) would adequately provide QoS control for many HSDPA RT and NRT multi-class session scenarios, due to the static prioritization of RT packets' transmission, occasions may arise where stalling of the NRT flow occurs 
at the radio link buffer. During heavier downlink shared channel loads or deteriorating UE channel conditions, potential NRT flow stalling is even more likely (thus annulling the gain of Iub flow control). A possible alleviating solution is to incorporate dynamic transmission priority switching between the concurrent RT and NRT flows. Hence, we propose a Dynamic Time-Space priority buffer management scheme to this effect. The D-TSP scheme is illustrated in Figure 11.

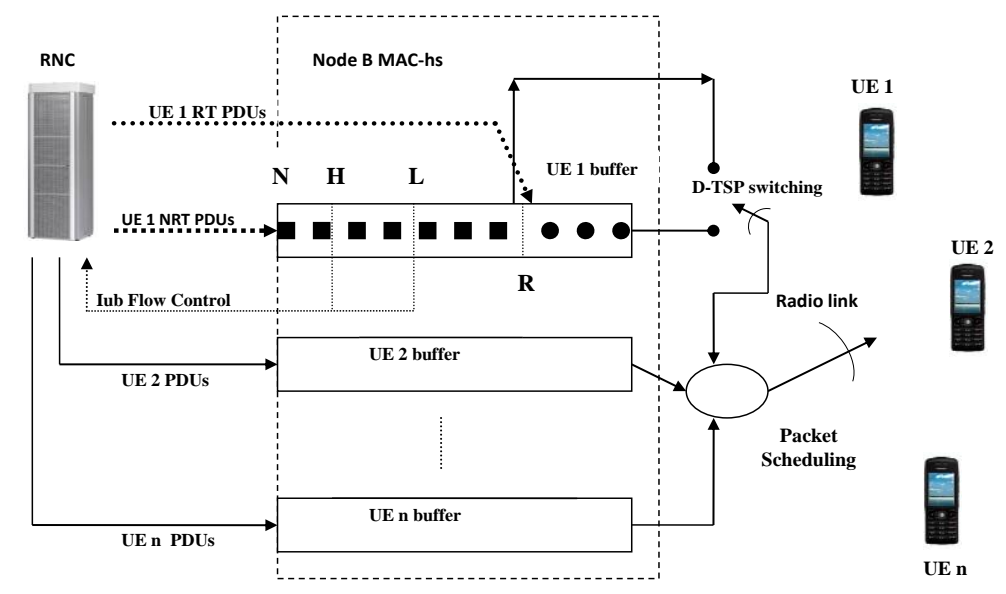

Figure 11: Dynamic Time-Space priority buffer management scheme in HSDPA Node B UE buffer.

D-TSP buffer management incorporates dynamic time priority switching with Iub flow control and TSP buffer admission control strategy. Basically, for a given transmission opportunity assigned to the UE by the Packet Scheduling discipline, when the headof-the-line RT packet queuing delay is below a given delay budget, transmission priority is switched to the NRT flow, otherwise it remains with the RT flow. Adopting the same notations and assumptions for E-TSP in 4.1, the further assumptions for D-TSP are as follows:

- We assume a given delay budget, DB, for the UE RT flow PDU queuing in the Node B MAC-hs. DB is used to determine the priority switching control parameter. RT flow PDU inter-arrival time, $i$ can be estimated from the already known GBR thus:

$$
i=\text { PDU_size }(\text { bits }) / \lambda_{\text {rt }}(\text { bits/sec) }
$$

- Define a priority switching control parameter $\delta$ given by:

$$
\delta=\mathrm{DB} / i
$$

- Assuming a discard timer (DT) [37] is used to discard MAC-d PDUs of the RT flow with Node B MAC-hs queuing delay exceeding a given maximum delay budget $\mathrm{DB}_{\max }$. If $\mathrm{Y}$ is the maximum allowable downlink delay then:

$\mathrm{DB}_{\max }=\mathrm{Y}-($ external network $+\mathrm{CN}+\mathrm{RNC}+\mathrm{Iub})$ delay

With the above assumptions and notations, the D-TSP scheme operates as follows with part 1 and part 2 exactly the same as in E-TSP with part 3 replaced by the following:

\section{Part 3: Dynamic Transmission priority control:}

- For each $\mathrm{UE}_{\mathrm{n}}$ transmission opportunity:

IF $\left(\mathrm{r}(\mathrm{t})<\delta\right.$ AND RT HOL delay $<\mathrm{DB}_{\max }$ AND $\left.\mathrm{n}(\mathrm{t})>0\right)$

$$
\{
$$

Time Priority $=$ NRT flow

Generate MAC-hs Transport Block from NRT PDUs

$$
\text { \} }
$$

ELSE

$$
\{
$$

Time Priority $=R T$ flow 
\}

\section{E-TSP and D-TSP END-TO-END PERFORMANCE EVALUATION}

In order to evaluate the end-to-end performance of the proposed TSP-based buffer management schemes, a custom system-level HSPDA simulator was developed using OPNET modeler. Simulation was chosen for the end-to-end performance evaluation rather than analytical modelling, in order to include as much system detail as possible to capture the dynamics of the scenarios more realistically. The simulation model is shown in Figure 12.

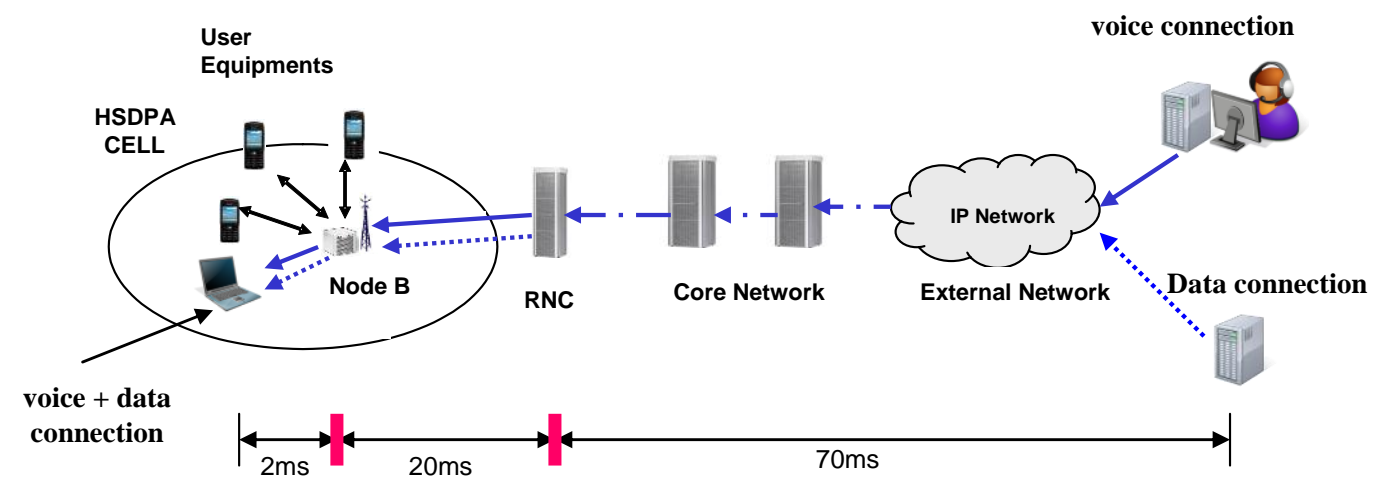

Figure 12. HSDPA Simulation model for E-TSP/D-TSP end-to-end performance evaluation.

The multi-class traffic sources comprise implementation of VoIP ON/OFF source with the same parameters employed by Bang et. al in [38], and a customizable NRT traffic source with TCP Reno implementation. Detailed modelling of the HSDPA data flow mechanisms and the entities involved as described in section 2.2 are included in the HSDPA simulator, except for the external network and core network elements, whose effects (as shown in Figure 12) are abstracted by a single assumed fixed delay of 70ms to packets arriving at the HSDPA RNC. The duration of the VoIP ON/OFF periods is negative exponentially distributed with mean duration of 3 seconds. The VoIP packet length is taken as 304 bits or 38 bytes including RTP/UDP/IP and RLC header overheads. Packets are assumed to arrive from the core network to the radio access network without any loss. For the Radio Access Network, a single HSDPA cell served by a Node B under the control of an RNC with the concurrent VoIP and FTP downlink traffic towards an end user (UE) was modelled. Radio propagation was modeled using ITU R path loss model and lognormal shadowing with std. deviation $\sigma=8 \mathrm{~dB}$. The path loss model used is taken from [39]:

$\mathrm{L}=40 \log _{10} R+30 \log _{10} f+39$

where $f$ is the WCDMA carrier frequency i.e. $2 \mathrm{GHz}$, and $R$ is the distance of the mobile from the base station in kilometers. The lognormal shadowing in logarithmic scale is characterized by a Gaussian distribution $N(0, \sigma)$ with Gaussian random variable of mean 0 and $\sigma=8$ [40]. The shadowing value is updated every $2 \mathrm{~ms}$. Due to the slow fading process versus distance, adjacent shadow fading values are correlated [41]. Thus the following correlation model is considered for shadow fading [39]:

$\mathrm{C}(d)=2^{-d / d c o r}$

where $\mathrm{C}(d)$ is a normalized autocorrelation function of shadowing when decorrelation length is given by dcor and the moving distance of the mobile after the last calculation of shadowing is $d$. Basically, the shadowing effect is represented by [39]:

$$
S=\mathrm{C}(d) * S^{\prime}+1-[\mathrm{C}(d)]^{2} * N(0, \sigma)
$$

$S$ is the shadowing value in $\mathrm{dB}$ updated with the last calculated value $S^{\prime}$. In the simulation $d$ cor is set to 5 meters [41].

Total Node B transmission power is assumed to be $15 \mathrm{~W}$, while the allocated HS-PDSCH power was 7W. The instantaneous throughput towards the UE is determined by transport block size (TBS) which is governed by the Adaptive modulation and coding 
(AMC) functionality. We assumed that six AMC schemes are available and selected based on the reported channel quality (the instantaneous Signal-to-Interference Ratio, SINR) of each UE. Table 2 shows the equivalent number of bits transmitted per HSPDSCH code per TTI for each of the AMC scheme. In the simulator, a look up table is used to map instantaneous SINR values to each of the AMC schemes in order to model the HSDPA CQI operation.

Table 2: Assumed AMC schemes and equivalent instantaneous bit rates per TTI [29]

\begin{tabular}{|c|c|}
\hline Modulation and Coding scheme & No of bits per code per TTI \\
\hline QPSK 1/4 & 240 \\
\hline QPSK 1/2 & 360 \\
\hline QPSK 1/3 & 480 \\
\hline QPSK 3/4 & 720 \\
\hline 16QAM 1/4 & 960 \\
\hline 16QAM 3/4 & 1440 \\
\hline
\end{tabular}

Due to channel quality transmission latency, packets may be received in error as it is possible for the UE SINR at that instance to be different from the last known SINR in the Node B that was used for AMC scheme selection. Thus HARQ re-transmission is modeled with soft combining of all received packets, with the effective SINR taken as $\boldsymbol{N} \cdot \boldsymbol{S N I R}$ init where N is the number of transmissions and SINR $\boldsymbol{R}_{\text {init }}$ is the SINR of the first transmission. MAC-d PDU size length after RLC packet segmentation/assembly is taken as 320 bits for both VoIP and TCP-based FTP flows in the multimedia traffic. In all the experiments, a test user equipment (UE 1) was assumed to be receiving multi-class traffic of simultaneous RT (VoIP) and NRT (FTP) during a 120s simulated voice conversation and file download session. VoIP packets were being received while file download was taking place using FTP over TCP. The overall set up models a single HSDPA cell with inter-user packet scheduling to $m$ users. A summary of the HSDPA parameters used are given in Table 3.

Table 3: Summary of assumed HSDPA simulation parameters for E-TSP and D-TSP end-to-end performance evaluation.

\begin{tabular}{|l|l|}
\hline \multicolumn{2}{|c|}{ HSDPA Simulation Parameters } \\
\hline Parameter & \multicolumn{1}{c|}{ Value } \\
\hline HS-DSCH TTI & $2 \mathrm{~ms}$ \\
\hline Path loss Model & $148+40 \mathrm{log}(\mathrm{R}) \mathrm{dB}$ \\
\hline Transmit powers & Total Node B power=15W, HSDSCH power= 50\% \\
\hline Shadow fading & Log-normal: $\sigma=8 \mathrm{~dB}$ \\
\hline AMC schemes & QPSK $1 / 4$, QPSK $1 / 2$, QPSK $3 / 4,16$ QAM $1 / 4,16$ QAM $1 / 2$ \\
\hline No. of assigned HSDSCH codes & 5 \\
\hline CQI delay & $3 \mathrm{TTIs}(6 \mathrm{~ms})$ \\
\hline HARQ processes & 4 \\
\hline HARQ feedback delay & $5 \mathrm{~ms}$ \\
\hline Test UE position from Node B & $0.2 \mathrm{~km}$ \\
\hline Packet Scheduling & Fair time \\
\hline MAC PDU size & $320 \mathrm{bits}$ \\
\hline Iub (RNC-Node B) delay & $20 \mathrm{~ms}$ \\
\hline External + CN delays & $70 \mathrm{~ms}$ \\
\hline HS-DSCH frame & $10 \mathrm{~ms}$ \\
\hline Flow control parameters & $\alpha=0.7 ; w=0.7 ; \mathrm{k}=0.5$ \\
\hline TCP Parameters: & Reno; MSS = 536 bytes; RWIND = 64 (32KB) \\
\hline
\end{tabular}

\subsection{E-TSP End-to-end QoS Performance Evaluation}

E-TSP end-to-end performance evaluation using the HSDPA simulator is discussed in this section. In order to determine if performance gains accrue from the proposed flow control algorithm and its effectiveness in mitigating buffer overflow, E-TSP is 
compared with (drop-tail) TSP queuing and CBS scheme (with FIFO service) as baseline schemes. Buffer scheme configurations are: $\mathrm{N}=150$, for CBS; $\mathrm{R}=10, \mathrm{~N}=150$, for TSP; $\mathrm{R}=10, \mathrm{~L}=30, \mathrm{H}=100, \mathrm{~N}=150$ for E-TSP. Various HSDPA channel loads are considered and a test UE assumed to be receiving VoIP and FTP downloads simultaneously, is observed for 120s. The performance metrics recorded include:

- End-to-end NRT throughput: the end-to-end file download TCP throughput at the test UE receiver during the concurrent VoIP and file download multi-class traffic session.

- End-to-end VoIP delay: The end-to-end delay of VoIP packets measured in the multi-class traffic test UE receiver during the concurrent VoIP and file download session.

\subsubsection{NRT flow end-to-end performance}

Figures 13-15 show results of the end-to-end TCP average throughput measurements with time, for the test UE receiving concurrent VoIP and FTP flows during the 120s multi-class session under various HSDPA cell loads. The test UE is assumed to be located $200 \mathrm{~m}$ away from the base station and moving away at $3 \mathrm{~km} / \mathrm{h}$ speed, while other UEs are placed at random positions in the cell and assumed to each be receiving a single flow of FTP downloads during their sessions and hence no buffer management scheme is applied to their MAC-hs queues.

Figure 13 plots the NRT throughput obtained with complete buffer sharing used in the MAC-hs for the multi-class traffic of the test UE; where arriving NRT or RT PDUs from the RNC for the test UE are queued and transmitted in a FIFO manner in an allocated buffer of capacity N. With more users scheduled on the HSDPA channel, the throughput observed in the test UE drops as expected. Since fair time scheduling is used, the throughput of the test UE is expected to drop with more users as the end-toend TCP RTT increases due to increased inter-scheduling gaps. Due to lack of flow control mechanism CBS is not immune from buffer overflow, but is generally expected to give better NRT throughput than TSP (with drop-tail) because of the fairer chance of access to transmission opportunity to NRT packets inherent in the FCFS service. The unsteadiness in the throughput over time can be attributed to loss recovery at the RLC layer when MAC-hs buffer overflows, and also possible loss recovery at the TCP layer in the event of RLC recovery failure (after a maximum of six attempts).

The same experiment is repeated with drop-tail TSP applied to the MAC-hs buffer of the test UE and the results are shown in Figure 14. Like CBS, TSP is also not immune from buffer overflow, so a similar pattern to Figure 13 is observed. While the average throughput is steady at almost $240 \mathrm{kbps}$ for the single user scenario, the throughput drops dramatically in the scenarios with more users on the shared channel. Also, the fact that some unsteadiness in the throughput is observed indicates that interscheduling gaps due to higher channel loading alone is not responsible for the drop, but buffer overflow (and consequent RLC and TCP loss recoveries) is a contributing factor.

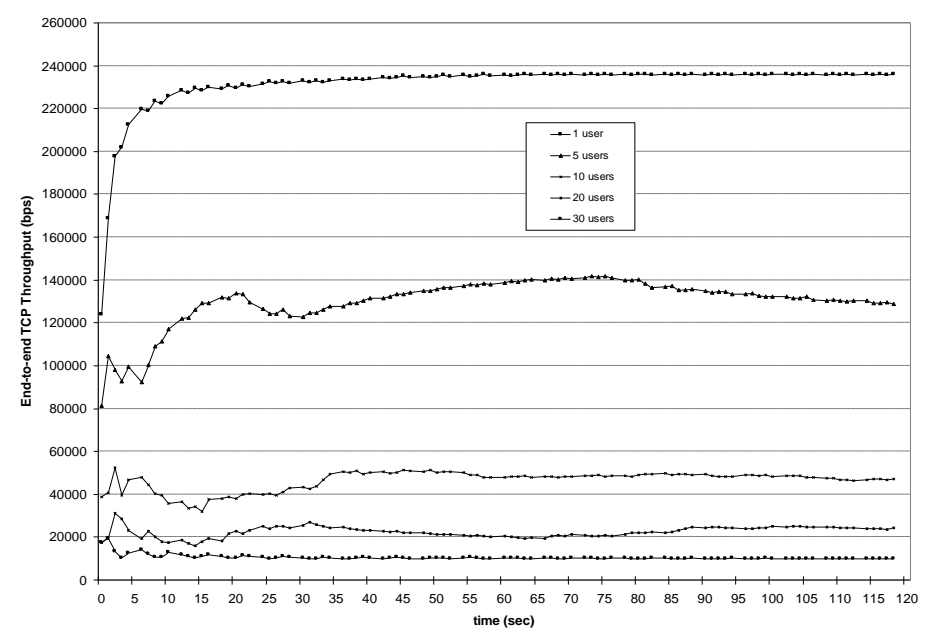

Figure 13: End-to-end NRT throughput at multimedia test UE with Complete Buffer Sharing for 1, 5, 10, 20 and 30 users utilizing the HSDPA shared channel. 




Figure 14: End-to-end NRT throughput at multimedia test UE with (drop-tail) TSP buffer management for 1, 5, 10, 20 and 30 users utilizing the HSDPA shared channel.

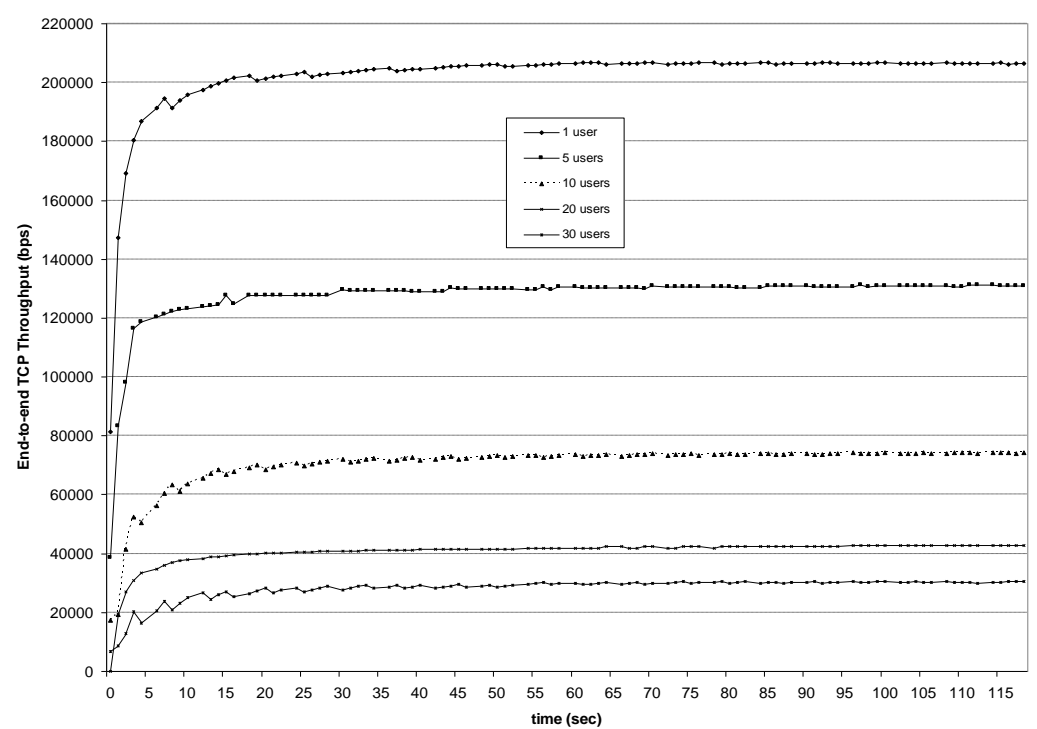

Figure 15: End-to-end NRT throughput at multimedia test UE with E-TSP buffer management for 1, 5, 10, 20 and 30 users utilizing the HSDPA shared channel.

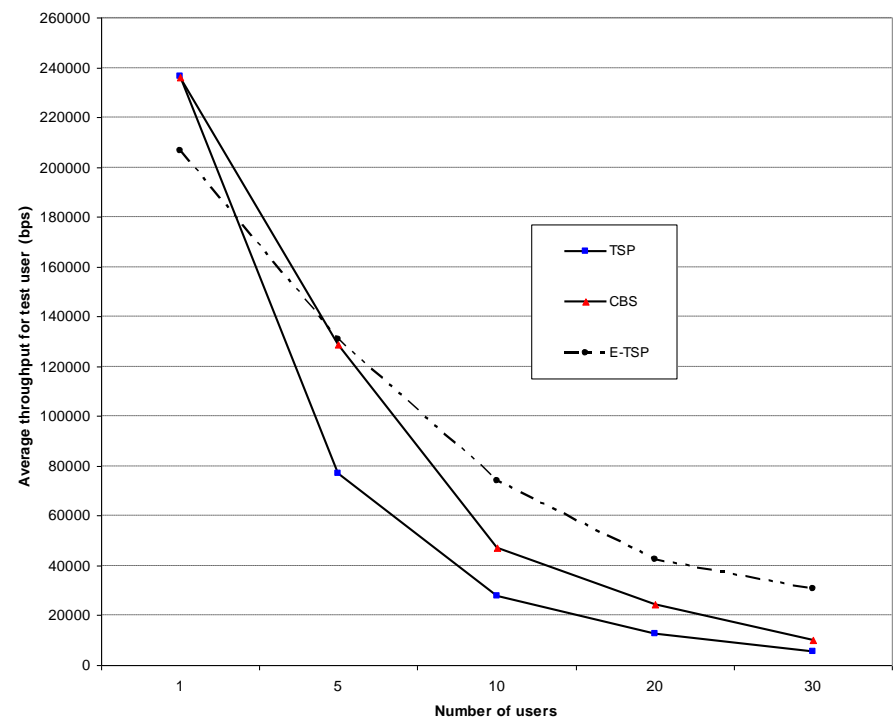

Figure 16: Average end-to-end NRT throughput at test UE Vs no. of users (CBS, TSP and E-TSP). 
In Fig. 15, results of the same experiment with the E-TSP scheme applied to the MAC-hs queue of the multi-flow user is shown. The test UE TCP throughput is seen to have a steady throughput after the initial rise, indicating comparatively lower RTT variation. This is because of lack of buffer overflow which prevents consequent RLC or TCP layer loss recovery. Thus, E-TSP was able to mitigate MAC-hs NRT PDU losses, reducing the occurrence of RLC level and hence TCP retransmissions. Of course the inevitable drop in throughput with more users can only be attributed to fewer scheduling opportunities and hence larger transmission gaps, but the flow control algorithm of E-TSP reacts accordingly to prevent buffer overflow.

Figure 16 illustrates the average NRT TCP throughput over the entire session for the three schemes, in a single graph. It shows that E-TSP yields significant throughput improvement especially with higher cell loads. It is interesting to observe from Figure 16 that E-TSP gave the lowest throughput for the single user scenario. The reason for this is the increased TCP RTT as a result of the flow control mechanisms and the time priority of the RT PDUs. But as the cell load increases, the effect of the inter-scheduling gaps and potential losses due to MAC-hs buffer overflow become the dominating factors. Since the E- TSP is equipped with the flow control mechanism, it is best able to cope with load and channel quality variation so that performance improvement is noticeable at higher load.

Note that the better NRT throughput performance that CBS achieves compared to drop-tail TSP is at the expense of RT flow QoS degradation (see Figure 17). That is, with CBS, NRT packets have fairer chance of scheduling opportunity at the expense of VoIP delay. On the other hand, the trade-off in NRT throughput as a result of TSP schemes always manifests in better VoIP delay performance (as is evident from Figure 17). One important implication of this set of results is that TSP-based schemes will benefit from additional mechanisms that can provide more fairness in NRT flow transmission bandwidth allocation at the expense of further trade-off in RT QoS performance as long as this is kept within maximum allowable constraints.

\subsubsection{RT flow end-to-end performance}

The most important observation to be made from Figure 17 is that the end-to-end VoIP QoS is not degraded as a result of the ETSP flow control scheme. Whereas, we observe from Figure 16 the corresponding end-to-end NRT performance gain with E-TSP. While TSP and E-TSP show identical VoIP performance in the multi-flow session because both have the time priority mechanism, they also maintain a fairly low variation in end-to-end delay with increasing load, compared to CBS. Thus, the set of results obtained demonstrate that E-TSP is an effective buffer management scheme for joint QoS control in multi-class sessions with RT and NRT flows, which when implemented at the radio link buffer can improve end-to-end traffic QoS of both flows. This idea is exploited in the D-TSP scheme whose performance is evaluated in section 5.2.

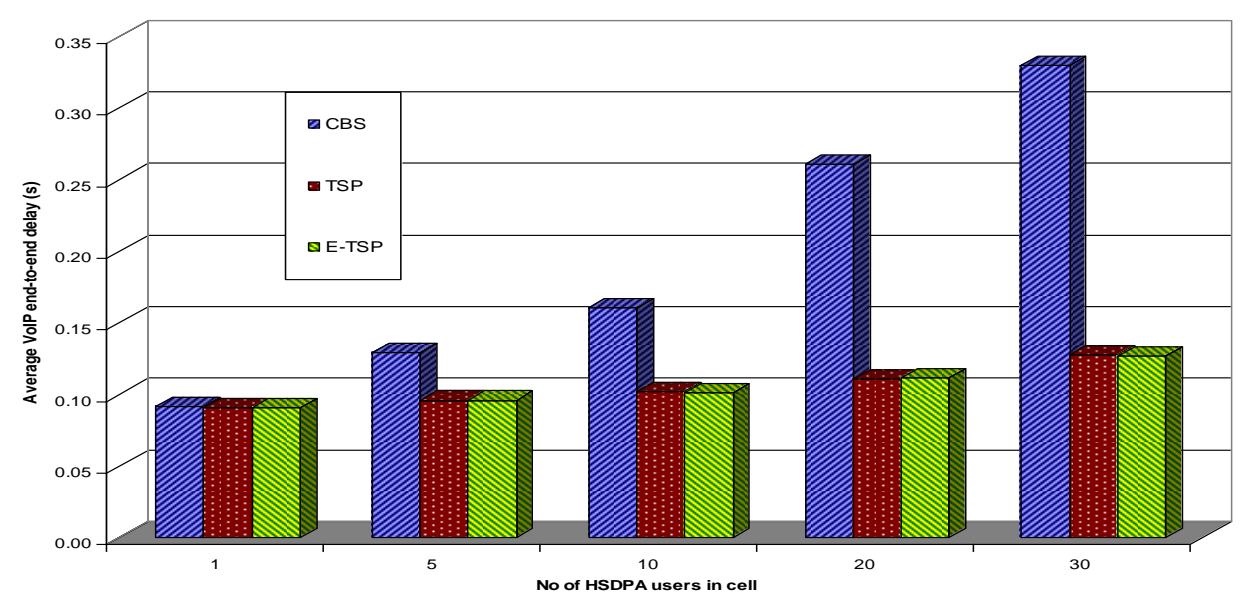

Figure 17: Average end-to-end VoIP PDU delay at test UE vs. number of users in cell. Results given for CBS (left) TSP (centre) and E- TSP. 


\subsection{D-TSP End-to-end QoS Performance Evaluation}

As mentioned earlier, D-TSP is proposed to ameliorate any potential NRT starvation problems in E-TSP due to static RT transmission prioritization, by incorporating dynamic priority switching, at the expense of slight RT QoS degradation. Hence, in the performance study we focus on potential end-to-end NRT throughput improvement with the dynamic priority switching and the impact on the RT flow QoS. We will therefore use the E-TSP scheme, which has static RT transmission prioritization, as the baseline scheme for comparative analyses. In the experiments, a test user equipment (UE 1) was assumed to be receiving multiflow traffic of simultaneous RT (VoIP) and NRT (FTP) during a 120s simulated voice conversation and file download session. VoIP packets were being received while file download was taking place using FTP over TCP. The overall set up models a single HSDPA cell with fair time scheduling to $\boldsymbol{m}$ users. The HSDPA simulation parameters used are those given in Table 3 . Buffer scheme configuration include : R=10 PDU, L=100 PDU, H=150 PDU, N = 200 PDU for both E-TSP and D-TSP.

We assume that maximum allowable one way VoIP delay is $250 \mathrm{~ms}$ [42], [43]. For the VoIP flow, we assume RNC queuing delay is negligible since the flow control algorithm is expected to ensure prompt transmission to the Node-B. Moreover, this was confirmed during the simulations. Hence, the maximum allowed queuing delay budget $\mathrm{D}_{\max }$, can be determined from equation (7) thus: $250-($ External $+\mathrm{CN}$ delays $)-$ Iub delay $=160 \mathrm{~ms}$. We therefore set the discard timer (DT) to 160ms. In the experiments, we consider MAC-hs queuing delay budgets, DB, of 40, 80, 120 and $160 \mathrm{~ms}$ which from equation (6), correspond to $\delta=2,4,6$ and 8 respectively, since VoIP PDU inter-arrival time is approximately $20 \mathrm{~ms}$ during ON periods given that $\lambda_{\mathrm{rt}} \approx 16 \mathrm{kbps}$ [38]. Note that the discard timer, DT discards VoIP packets whose MAC-hs queuing delay $\geq 160 \mathrm{~ms}$ from the head of the D-TSP queue. DT is also used with the E-TSP scheme. The performance metrics observed include:

- End-to-end NRT throughput: the end-to-end TCP throughput at the test UE 1 during file download in the multi-class session.

- $\quad$ RT PDU Discard Probability: defined as the number of late HOL RT PDUs discarded from the (D-TSP or E-TSP) MAC-hs queue as a result of DT timeout.

- Percentage air interface utilization: calculated from Transport Block Size transmitted divided by maximum Transport Block Size allowable by the selected AMC scheme, measured at every transmission opportunity.

\subsubsection{NRT flow end-to-end performance}

Figure 18 shows the average end-to-end NRT flow TCP throughput of the test UE running a VoIP and FTP multi-class session under various channel loads. The test UE is assumed to be located at $0.2 \mathrm{~km}$ from the Node B and moving away at $3 \mathrm{~km} / \mathrm{h}$ speed. We see that in the 1 user scenario, the D-TSP scheme in all DB (delay budget) settings give identical average throughput to ETSP. This represents a lightly loaded HSDPA channel where all the channel resources are allocated to the test UE at every TTI resulting in high bandwidth allocation. Under this condition, application of D-TSP with even the most relaxed DB setting can only yield very marginal NRT throughput improvement. On the other hand, as the load is increased, D-TSP gives higher average endto-end throughput especially with higher DB settings. As the cell becomes more heavily loaded the radio-link 'bottleneck' effect worsens and end-to-end TCP throughput generally drops, but in all cases throughput gains are achieved with D-TSP. 


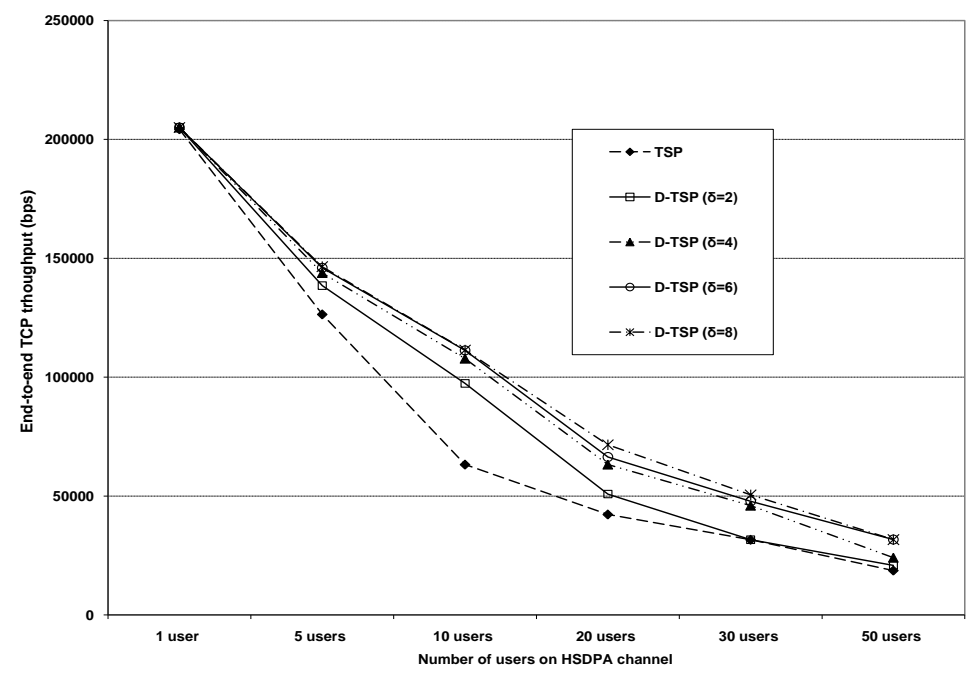

Figure 18: End-to-end NRT throughput of test UE for E-TSP, DTSP $(\delta=2,4,6,8)$ with 1, 5, 10, 20,30 and 50 users sharing the HSDPA channel.

\subsubsection{RT flow end-to-end performance}

Since a Discard Timer is used to discard Head-of-Line VoIP PDUs with delay exceeding the discard time DT setting of 160ms, PDUs violating the delay deadline bound will not be received at the test UE 1. Thus, as a measure of the UE 1 VoIP QoS in the multi-class session, we consider the VoIP PDU discard probabilities for the aforementioned scenarios for both TSP and D-TSP with the various DB settings. The results are illustrated in Figure 19. Generally, more VoIP PDUs are discarded from the UE 1 MAC-hs queue as more users are scheduled on the HSDPA channel, and also with higher DB settings which correspond to more relaxed delay budget. Assuming a maximum of discard ratio of $2 \%$ is acceptable for VoIP QoS, Figure 19 shows that VoIP QoS is satisfied in all cases of D-TSP and E-TSP for the 1-user, 5-user, 10-user scenarios (Recall that the discard timer DT mechanism is also applied in E-TSP). Whereas, for the 20-user scenario, the maximum setting of $\delta$ for D-TSP is 6 corresponding to maximum delay budget of $120 \mathrm{~ms}$. For the 30 -user scenario, maximum acceptable $\delta=4$ corresponding to maximum delay budget of $80 \mathrm{~ms}$. While in the 50-user scenario, maximum acceptable setting for $\delta$ is 2 corresponding to maximum delay budget of $40 \mathrm{~ms}$.

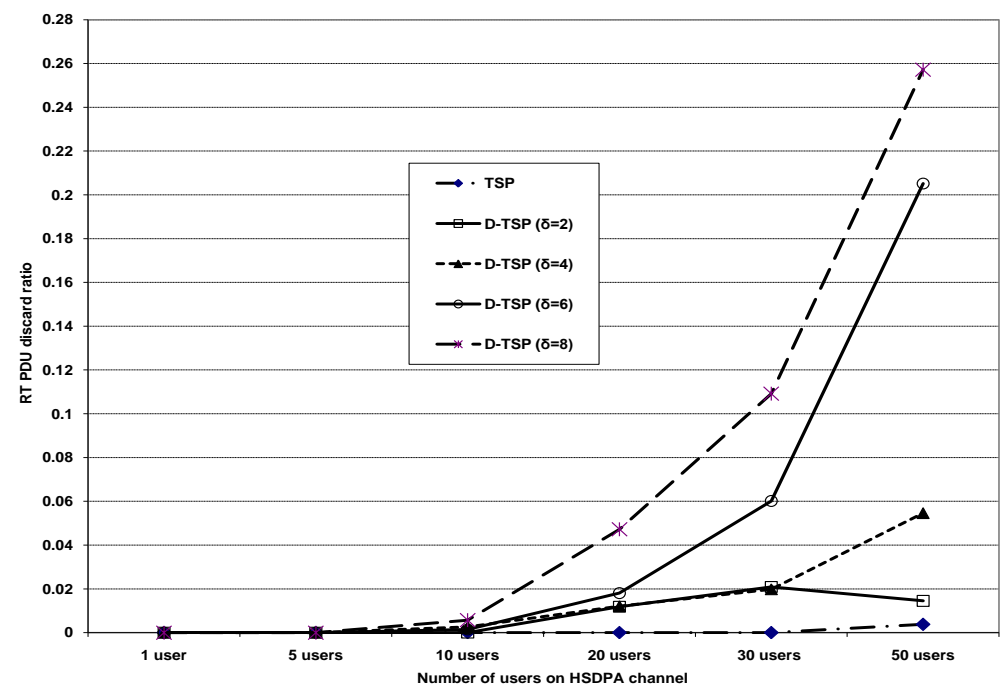

Figure 19: UE 1 VoIP PDU Discard probability for vs. number of HSDPA users.

\subsubsection{HSDPA channel utilization}

In addition to the throughput performance gain, D-TSP also improves the air interface utilization on the HSDPA channel compared to E-TSP. As seen from Figure 20, the higher the number of users being scheduled on the air interface with the better the air interface utilization. For instance, in the 20-user scenario, total channel utilization is $54 \%$ for both RT and NRT flows in the multi-flow session of the UE 1 when using TSP. With D-TSP and $\delta=6$, on the other hand, utilization of almost $62 \%$ is 
achieved. This is due to VoIP PDU bundling in the Transport Block during transmission. The fact that increased utilization of the radio resources is more noticeable with higher $\delta$ for the higher cell loading scenarios reflects the statistical multiplexing properties of the HSDPA high-speed downlink shared channel (HS-DSCH)

The set of results presented in this section demonstrate that D-TSP can achieve better radio link bandwidth fairness to improve NRT end-to-end throughput by exploiting RT flow tolerance within allowable QoS constraints. The scheme is capable of optimizing the QoS performance between the two classes of flows during the UE session. With the improved bandwidth fairness due to dynamic priority switching, potential NRT bandwidth starvation problem is eliminated.



Figure 20: UE 1 HSDPA channel utilization for the various D-TSP settings.

\section{Concluding remarks}

This paper presented two novel buffer management schemes, E-TSP and D-TSP, for HSDPA radio link QoS control of concurrent RT and NRT flows transmitted to the same user. The schemes are based on time-space priority queuing (TSP) system which provides time priority with precedence queuing and restricted admission for RT class, while NRT class is queued with unrestricted buffer access but lower transmission priority. TSP was compared to conventional queuing schemes like CBS, PBP, and PBS using Markov models, and the results illustrate the effectiveness of TSP queuing in joint RT and NRT QoS control in the multi-class traffic in addition to efficient buffer utilization. Furthermore, using extensive system-level HSDPA simulations, we showed that E-TSP can achieve end-to-end NRT throughput performance gains without compromising RT QoS by preventing buffer overflow. Finally, also via extensive HSDPA system simulations, we demonstrated the radio link bandwidth allocation fairness and resulting end-to-end NRT performance improvement achievable with dynamic time priority switching in D-TSP, besides its capability for optimum RT and NRT QoS control. Although the proposed schemes were proposed for and evaluated in HSDPA multimedia traffic scenarios, the concepts can be extended to other wireless systems with multi-class queuing at the air interface. In further work, the impact of the proposed buffer management schemes on system capacity, i.e. impact on performance of QoS-aware call admission control and handoff algorithms will be investigated.

\section{Acknowledgement}

The authors are grateful for the support of Higher Education Council for Wales (HFCE) ORSAS scheme in the duration of this work. 


\section{References}

[1] H. Holma and A. Toskala, WCDMA for UMTS-HSPA Evolution and LTE: John Wiley \& Sons Ltd, 2007.

[2] 3GPP, "High Speed Downlink Packet Access; Overall Description," 3GPP TR 25.308 version 5.7.0, December 2004.

[3] A. Golaup, O. Holland, and A. H. Aghvami, "Concept and Optimization of an effective Packet Scheduling Algorithm for Multimedia Traffic over HSDPA," in IEEE International Symposium on Personal, Indoor and Mobile Radio Communications. Berlin, Germany, 2005.

[4] L. Chuang and L. Yin., "Dynamic partial buffer sharing scheme: Proportional packet loss rate," in Proceedings of ICCT2003, 2003, pp. 259-262.

[5] J. W. Causey and H. S. Kim, "Comparison of buffer allocation schemes in ATM switches: Complete sharing, partial sharing, and dedicated allocation," in Proceedings of International Conference on Communications, vol. 2, May 1994, pp. 1164-1168.

[6] A. Y. M. Lin and J. Silvester, "Priority Queuing Strategies and Buffer Allocation Protocols for Traffic Control at an ATM Integrated Broadband Switching System " IEEE Journal on Selected Areas in Communications, vol. SAC-9, No. 9, pp. 1524- 1536, December 1991.

[7] A. K. Choudhury and E. L. Hahne, "Space priority management in a shared memory ATM switch " in Proc. IEEE GLOBECOM 93, vol. 3, 1993, pp. 1375-1383.

[8] T. Czachovski and F. Peregrine, "A queuing model for optimal control of partial buffer sharing," ATM Computer Operation Research, vol. 25 (2), pp. 113-126, Feb. 1998.

[9] C. G. Kang and H. H. Tan, "Queuing analysis of explicit priority assignment partial buffers sharing schemes for ATM networks " in Proc. IEEE/ACM INFOCOM, March 1993, pp. 810-819.

[10] J. J. Bae, T. Suda, and R. Simha, "Analysis of a Finite Buffer Queue with Heterogeneous Markov Modulated Poisson Arrival Processes: A Study of Traffic Burstiness and Priority Packet Discarding," in Proc. IEEE INFOCOM '92, August 1992.

[11] K. Bala, I. Cidon, and K. Sohraby, "Congestion Control for High Speed Packet Switched Network," in Proc. IEEE INFOCOM '90, June 1990, pp. 520-526.

[12] H. Kroner, G. Herbuterne, P. Boye, and A. Gravey, "Priority Management in ATM Switching Nodes," IEEE Journal on Selected Areas in Communications, vol. 9, pp. 418-427, April 1991

[13] G. Gallassi, G. Rigolio, and L. Fratta, " Bandwidth Assignment in Prioritized ATM Networks," in Proc.IEEE GLOBECOM '90, December 1990 pp. 852-856.

[14] H. Kroner, "Comparative Performance Study of Space Priority Mechanisms for ATM Networks " in Proc. IEEE INFOCOM '90, June 1990, pp. 1136-1143.

[15] G. Hetbuterne and A. Gravey, "A Space Priority Queueing Mechanism for Multiplexing ATM Channels," Computer Networks and systems, pp. 37-43, 1990.

[16] D. Mitra and I. Ziedins, "Virtual partitioning by dynamic priorities: Fair and efficient resource-sharing by several services, ," in Broadband Communications: Network Services, Applications, Future Directions, Lecture Notes in Computer Science: Spinger Verlag, 1996, pp. 173-185.

[17] J.-W. Cho and H. Dong, "Dynamic buffer management scheme based on rate estimation in packet-switched networks," Computer Networks, vol. 39, No.6, pp. 769-787, August, 2002.

[18] I. Cidon, L. Georgiadis, R. Guerin, and A. Khamisy, "Optimal Buffer Sharing," IEEE J. Select. Areas Commun., vol. 13, pp. 12291240, Sept. 1995.

[19] I. Awan and K. Al-Begain, "An Analytical Study of Quality of Service Provisioning for Multi-service Mobile IP Networks Using Adaptive Buffer Management," in Proc. of 11th International Conference on Analytical and Stochastic Modelling Techniques and Applications (ASMTA04). Magdeburg, Germany, June 2004, pp. 166-172.

[20] S. Ahmad, I. Awan, and B. Ahmad, "Performance Modelling of Finite Capacity Queues with Complete Buffer Partitioning Scheme for Bursty Traffic " in Proc. First Asia International Conference on Modelling and Simulation (AMS'07). Phuket, Thailand, March 2007.

[21] T. E. Kolding, "Link and System Performance Aspects of Proportional Fair Packet Scheduling in WCDMA/HSDPA," in Proc. IEEE Vehicular Technology Conference, Sept. 2003, pp. 1717-1722.

[22] G. Barriac and J. Holtzman, "Introducing Delay Sensitivity into the Proportional Fair Algorithm for CDMA Downlink Scheduling," in IEEE Proc. International Symposium on Spread Spectrum Techniques and Applications, September 2002, pp. 652-656.

[23] P. A. Hosein, "QoS Control for WCDMA High Speed Packet Data," in IEEE Proc. Vehicular Technology Conference, 2002.

[24] O. Holland, A. Golaup, and H. Aghvami, "Efficient Packet Scheduling for HSDPA Allowing Inter-class Prioritization," IET Electronics Letters, vol. 42. No. 18, Aug 2006.

[25] K. I. Pedersen, P. E. Mogensen, and T. E. Kolding, "QoS Considerations for HSDPA and Performance Results for Different Services," in Proc. IEEE 64th Vehicular Technology Conference VTC-2006 25-28 Sept. 2006, pp. 1-5.

[26] 3GPP, "Medium Access Control (MAC) protocol specification," 3GPP TS 25.321 version 7.5.0, June 2007.

[27] 3GPP, "Radio Link Control (RLC) protocol specification," TS 25.322 version 5.13.0, December 2005.

[28] 3GPP, "High Speed Downlink Packet Access : Iub/Iur protocol aspects," 3GPP TS 25.877 Version 5.4.0, Release 5, June 2002.

[29] H. Holma and A. Toskala, HSDPA/HSUPA for UMTS: John Wiley \& Sons Ltd, 2006.

[30] K. Al-Begain, A. Dudin, and V. Mushko, "Novel Queuing Model for Multimedia over Downlink in 3.5G Wireless Networks," Journal of Communications Software and Systems, vol. 2, No 2, June 2006.

[31] P. Wüchner, H. D. Meer, J. Barner, and G. Bolch, "A brief Introduction to MOSEL-2," in Proceedings of 13th GI/ITG Conference: Measuring Modeling and Evaluation of Computer and Communication Systems. Nürnberg, Germany, 2006, pp. 473-476.

[32] K. Al-Begain, J. Barner, G. Bolch, and A. I. Zriekat online, "The performance and Reliability Modelling Language MOSEL and its Application," International Journal of Simulation: Systems, Science and Technology vol. 3 No. 3-4, pp. 66-80, 2002.

[33] A. I. Zriekat, S. Y. Yerima, and K. Al-Begain, " Performance Evaluation and Resource Management of Hierarchical MACRO/MICRO Cellular Networks Using MOSEL-2," Wireless Personal Communications, vol. 44 , Issue 2 pp. 153 - 179 January 2008. 
[34] G. Ciardo, R. M. Fricks, J. Muppala, and K. S. Trivedi, "SPNP Users Manual Version 4.0," Duke University, Department of Electrical Engineering, Durham, NC. March 1994.

[35] G. Bolch, S. Greiner, H. D. Meer, and K. S. Trivedi, Queueing Networks and Markov Chains: John Wiley \& Sons Ltd., 1998.

[36] 3GPP, "UTRAN Iub Interface User Plane Protocols for Common Transport Channel data streams " 3GPP TS 25.435 Version 5.7.0, March 2004.

[37] K. I. Pedersen, P. E. Mogensen, and T. E. Kolding, "Overview of QoS Options for HSDPA," in IEEE Communications Magazine, July 2006, pp. 100-105.

[38] W. Bang, K. I. Pedersen, T. E. Kolding, and P. E. Mogensen, "Performance of VoIP on HSDPA," in Proc. IEEE VTC. Stockholm, June 2005.

[39] ITU, "Guidelines for Evaluation of Radio Transmission Technologies for IMT-2000," Recommendation ITU-R M.1225 1997.

[40] 3GPP, "Physical Layer Aspects of UTRA High Speed Downlink Packet Access (Release 4)," TR 25.848 version 5.13.0, March 2001.

[41] W. S. Jeong, D. G. Jeong, and B. Kim, "Packet Scheduler for Mobile Internet Services Using High Speed Downlink Packet Access," IEEE Transactions on Wireless Communications, vol. 3, No.5, September 2004.

[42] ITU, " One-way Transmission Time " ITU Recommendation G.114 2003.

[43] R. Cuny and A. Lakaniemi, "VoIP in 3G Networks: An end-to-end quality of service analysis," in Proc. IEEE Vehicular Technlogy Conference vol. 2, April 2003, pp. 930-934. 